\title{
Überzeugungen und Selbstwirksamkeitserwartungen zum inklusiven Unterricht bei (angehenden) Lehrkräften: der Einfluss von Lerngelegenheiten
}

\author{
Claudia Menge $\cdot$ Thorsten Euler $\cdot$ Hildegard Schaeper
}

Eingegangen: 25. Juni 2020 / Überarbeitet: 10. Februar 2021 / Angenommen: 19. April 2021 / Online publiziert: 13. August 2021

(C) Der/die Autor(en) 2021

Zusammenfassung Dieser Beitrag untersucht den Einfluss von Lerngelegenheiten und Unterrichtserfahrungen auf die inklusionsbezogenen Überzeugungen und Selbstwirksamkeitserwartungen von (angehenden) Lehrkräften. Die Daten stammen aus einer Onlinebefragung der Studierendenkohorte des Nationalen Bildungspanels (NEPS) im Herbst 2016. Das Analysesample umfasst Studierende, Referendarinnen und Referendare sowie erwerbstätige Lehrkräfte $(N=1862)$. Ein Strukturgleichungsmodell ergab, dass inklusionsbezogene Lerninhalte im Rahmen von Lehramtsstudium und Referendariat förderlich für die inklusionsbezogene Selbstwirksamkeitserwartung sind, während die Überzeugungen zu inklusivem Unterricht weitgehend unbeeinflusst bleiben. Ein Schulformvergleich zeigte, dass (angehende) Sonder-/ Förder- und Grundschullehrkräfte dem Unterrichten von inklusiven Schulklassen positiver und zuversichtlicher gegenüberstehen als (angehende) Lehrkräfte der Sekundarstufe I, Gymnasien und beruflichen Schulen. Zudem wurde festgestellt, dass

Hinweis Diese Arbeit nutzt Daten des Nationalen Bildungspanels (NEPS) Startkohorte Studierende, https://doi.org/10.5157/NEPS:SC5:11.0.0. Die Daten des NEPS wurden von 2008 bis 2013 als Teil des Rahmenprogramms zur Förderung der empirischen Bildungsforschung erhoben, welches vom Bundesministerium für Bildung und Forschung (BMBF) finanziert wurde. Seit 2014 wird das NEPS vom Leibniz-Institut für Bildungsverläufe e. V. (LIfBi) an der Otto-Friedrich-Universität Bamberg in Kooperation mit einem deutschlandweiten Netzwerk weitergeführt. Das dieser Arbeit zugrundeliegende Vorhaben wurde u. a. mit Mitteln des Bundesministeriums für Bildung und Forschung unter dem Förderkennzeichen B1014B gefördert. Die Verantwortung für den Inhalt dieser Veröffentlichung liegt bei den Autorinnen und Autoren.

Claudia Menge $(\bowtie) \cdot$ Thorsten Euler $\cdot$ Dr. Hildegard Schaeper

Deutsches Zentrum für Hochschul- und Wissenschaftsforschung (DZHW), Lange

Laube 12, 30159 Hannover, Deutschland

E-Mail: menge@dzhw.eu

Thorsten Euler

E-Mail: euler@dzhw.eu

Dr. Hildegard Schaeper

E-Mail: schaeper@dzhw.eu 
erwerbstätige Lehrkräfte trotz häufigerer Lerngelegenheiten eine kritischere und weniger zuversichtliche Haltung zu Inklusion einnehmen als Studierende oder Referendarinnen und Referendare.

Schlüsselwörter Lehrkräftebildung · Überzeugungen · Selbstwirksamkeit · Inklusiver Unterricht

\title{
Beliefs and perceived self-efficacy of (prospective) teachers regarding inclusive education: the effect of learning opportunities
}

\begin{abstract}
This paper examines the effects of learning opportunities and experiences on (prospective) teachers' beliefs towards inclusion and perceived self-efficacy regarding inclusive teaching. The data are taken from a web survey in autumn 2016 of the German National Educational Panel Study (NEPS), Starting Cohort "FirstYear Students". The sample comprises teacher students in different phases of teacher training and active teachers $(N=1862)$. The results of a structural equation model indicated that learning opportunities foster self-efficacy regarding inclusive classes while beliefs about inclusive teaching remain unaffected. The comparison of different types of schools showed that (prospective) teachers for special and primary education are more positive and confident about teaching inclusive classes, while (future) teachers for lower and upper secondary education are more critical. In addition, it could be observed that active teachers, despite higher levels of experience and learning opportunities, present themselves less positive and less confident about inclusive education than respondents who are still in teacher training.
\end{abstract}

Keywords Beliefs · Inclusive education · Self-efficacy $\cdot$ Teacher education

\section{Einleitung: Zunehmende Diversität im deutschen Regelschulsystem}

Auf Grundlage der Salamanca-Erklärung der UNESCO (UNESCO 1994), der Ratifizierung der UN-Behindertenrechtskonvention (United Nations 2006) und des Beschlusses der Kultusministerkonferenz zur inklusiven Bildung von Kindern und Jugendlichen mit Behinderungen in Schulen (Kultusministerkonferenz 2011) strebt die deutsche Bildungspolitik einen gleichberechtigten Zugang von Menschen mit Behinderungen zur schulischen Bildung an. Infolgedessen kam es in den letzten Jahren zu einem deutlichen Anstieg der Beschulung von Schülerinnen und Schülern mit sonderpädagogischem Förderbedarf (SPF) an Regelschulen von 12,4\% im Schuljahr 2000/01 (Autorengruppe Bildungsberichterstattung 2018) auf 42,3\% im Schuljahr 2018/19 (Autorengruppe Bildungsberichterstattung 2020). Die Inklusionsquote sinkt dabei von der Elementar- zur Primar- und Sekundarstufe, „d.h. alle Übergänge sind Schwellen in den Bildungsbiographien mit einem hohen Exklusionsrisiko", das insbesondere vom jeweiligen Förderschwerpunkt, z. B. der geistigen oder der körperlich/motorischen Entwicklung, abhängt (Eichfeld und Algermissen 2016, S. 68). Jedoch ist zu erwarten, dass der Anteil von Schülerinnen und Schü- 
lern mit SPF an Regelschulen weiter ansteigen wird und Lehrkräfte dort häufiger in inklusiven Settings unterrichten werden.

Lehrkräfte sind die wichtigsten Akteure bei der erfolgreichen Umsetzung inklusiver Bildung, da sie die Lernumwelt im Klassenzimmer maßgeblich beeinflussen. Es liegt in ihrer Verantwortung, den Unterricht und die Lehrkonzepte ganzheitlich an den spezifischen Bedürfnissen der Schülerinnen und Schüler zu orientieren und diese individuell zu fördern (Schwab und Seifert 2015). Entsprechende professionelle Kompetenzen der Lehrkräfte stellen daher wichtige Voraussetzungen für die erfolgreiche Implementierung von Inklusion (Hellmich und Görel 2014) und das Abfedern beruflicher Belastungen (Abele und Candova 2007) dar. Der Lehramtsausbildung kommt dabei eine Schlüsselrolle zu (Hochschulrektorenkonferenz und Kultusministerkonferenz 2015). Gemäß dem Modell zur professionellen Kompetenz von Lehrkräften von Baumert und Kunter (2006) umfassen diese Kompetenzen Professionswissen, Überzeugungen und Werthaltungen, motivationale Orientierungen und selbstregulative Fähigkeiten. Für die Umsetzung der Inklusion spielen - neben dem pädagogischen und fachdidaktischen Wissen als Facetten des Professionswissens - vor allem Überzeugungen der Lehrkräfte zum inklusiven Lernen eine maßgebliche Rolle (Hellmich und Görel 2014).

Aus diesem Grund nimmt dieser Beitrag inklusionsbezogene Überzeugungen und Selbstwirksamkeitserwartungen von (angehenden) Lehrkräften als Teil der professionellen Kompetenz in den Blick und fragt, inwieweit diese von Lerngelegenheiten $\mathrm{zu}$ inklusivem Unterricht beeinflusst werden. Im Unterschied zu vielen anderen Untersuchungen zu diesem Thema, die eine einzelne Schulform (z.B. Bosse et al. 2016; Eichfeld und Algermissen 2016; Hellmich und Görel 2016) oder eine spezifische Phase der Lehrkräftebildung bzw. -erwerbstätigkeit (z. B. Kopp 2009; Hellmich et al. 2016; Schwab und Seifert 2015) fokussieren, hat der hier verwendete Datensatz den Vorteil, alle (angestrebten) Lehrämter und Phasen der Lehrkräftebildung sowie die frühe Phase der Berufstätigkeit abzudecken. Damit verspricht der Beitrag, eine Forschungslücke zu schließen.

\section{Theoretischer Hintergrund, Forschungsstand und Hypothesen}

\section{1 Überzeugungen von Lehrkräften zur Inklusion}

Wie erwähnt spielen für die erfolgreiche Umsetzung inklusiver Bildung neben dem Professionswissen von Lehrkräften zu inklusiven Settings inklusionsbezogene Überzeugungen eine zentrale Rolle (Hellmich und Görel 2014). Theoretisch fundieren lässt sich diese Annahme mit den Überlegungen von Bandura (1997) zur Handlungsrelevanz von Selbstwirksamkeitserwartungen und dem Modell von Fives und Buehl (2012) zu den Funktionsweisen und Wirkungen von Überzeugungen. Empirisch ist die Befundlage noch dünn (Savolainen et al. 2020; Sermier Dessemontet et al. 2011), jedoch gibt es inzwischen einige Studien, die den Zusammenhang zwischen inklusionsbezogenen Überzeugungen und Selbstwirksamkeitserwartungen auf der einen Seite und effektivem Unterricht sowie inklusiven Lehrstrategien wie individualisiertem und differenziertem Unterricht auf der anderen Seite empirisch 
stützen (z. B. Hellmich et al. 2019; Jordan et al. 2009; Schwab et al. 2019; Wilson et al. 2016).

Das Konzept der Überzeugungen ist alles andere als einheitlich definiert und wird häufig synonym mit Begriffen wie Einstellungen, implizite oder subjektive Theorien, Haltungen oder Konzeptionen verwendet (Pajares 1992; Ruberg und Porsch 2017). Ungeachtet vielfältiger Überlappungen grenzen wir, Richardson (1996) und Fishbein (1967) folgend, Einstellungen von Überzeugungen durch das unterschiedliche Gewicht kognitiver und affektiver Bestandteile ab: Während sich Einstellungen in vielen Konzeptionen durch eine bewertende Komponente auszeichnen (vgl. die Definitionen von Eagly und Chaiken 1993; Fishbein 1967; Greiner et al. 2020; Greve und Hauenschild 2017), wird bei der begrifflichen Bestimmung von Überzeugungen der Fokus stärker auf kognitive Facetten gelegt. So definiert Richardson (1996, S. 104) Überzeugungen (,,beliefs“) als ,proposition that is accepted as true by the individual holding the belief" und Fishbein (1967) versteht Überzeugungen als Hypothesen über die Natur von Objekten.

Auch wenn ,there is no escaping the intertwined nature of knowledge and belief“ (Pajares 1992, S. 313), können Überzeugungen und Wissen u. a. aufgrund des unterschiedlichen epistemischen Status unterschieden werden (Pajares 1992; Richardson 1996; Steiger 2019). So impliziert Wissen eine epistemische Rechtfertigung (,epistemic warrant“; Richardson 1996, S. 104), es basiert auf einer „truth condition“ (Richardson 1996, S. 104), auf Evidenz, und auf einem Konsens hinsichtlich der Validität und Angemessenheit (Pajares 1992). Überzeugungen dagegen haben ,weder den Kriterien der Widerspruchsfreiheit noch den Anforderungen der argumentativen Rechtfertigung und der diskursiven Validierung zu genügen. Es genügt der individuelle Richtigkeitsglaube“ (Baumert und Kunter 2006, S. 497).

Diesen Ausführungen entsprechend können unter pädagogischen, bildungsbezogenen Überzeugungen von Lehrkräften ,,Vorstellungen und Annahmen von Lehrkräften über schul- und unterrichtsbezogene Phänomene und Prozesse“ (Kunter und Pohlmann 2015, S. 267) verstanden werden. Hinsichtlich der Überzeugungen zu Inklusion lassen sich dabei z. B. allgemeine Überzeugungen zum inklusiven Lernen, wie die Meinung zur allgemeinen Umsetzbarkeit der Inklusion von Schülerinnen und Schülern mit SPF an Regelschulen, sowie Überzeugungen zu spezifischeren Teilaspekten wie den Effekten inklusiven Lernens, der Ausgestaltung des Unterrichts oder der Zusammenarbeit mit Kolleginnen und Kollegen sowie Eltern unterscheiden.

Selbstwirksamkeitserwartungen können als eine spezifische Form von Überzeugungen, nämlich als Überzeugungen über das Selbst (,self-beliefs“), verstanden werden (Kunter und Pohlmann 2015; Pajares 1992). Der sozial-kognitiven Theorie Banduras (1997) folgend sind Selbstwirksamkeitserwartungen allgemein als der Glaube einer Person an ihre eigenen Fähigkeiten, auch schwierige Anforderungen oder Problemsituationen durch ihr eigenes Handeln bewältigen zu können, zu definieren. Kunter und Pohlmann (2015, S. 268) konkretisierten die Lehrerselbstwirksamkeitserwartung dementsprechend als „Überzeugungen einer Lehrperson darüber, wie gut es ihr gelingen kann, effektiv zu unterrichten“. Selbstwirksamkeitserwartungen in Bezug auf inklusiven Unterricht können dann mit Greiner et al. (2020, S. 276) als Überzeugung konzeptualisiert werden, ,Unterricht so gestalten zu können, dass 
Schülerinnen und Schüler mit und ohne sonderpädagogischen Förderbedarf gemeinsam an einem Lerngegenstand lernen können“".

Trotz der soeben erläuterten Definition von (Selbst-)Überzeugungen, die dieser Analyse zugrunde liegt, wird bei den nachfolgenden theoretischen Überlegungen und Erörterungen des Forschungsstands der konzeptionellen Nähe von Überzeugungen und Einstellungen Rechnung getragen und auch auf Arbeiten rekurriert, die inklusionsbezogene Einstellungen zum Gegenstand haben.

\subsection{Der Einfluss von Lerngelegenheiten auf Überzeugungen zur inklusiven Bildung}

Pädagogische Überzeugungen von (angehenden) Lehrkräften bilden sich im Allgemeinen sehr früh vor allem aufgrund von Erfahrungen in der eigenen Schulzeit heraus (Pajares 1992; Richardson 1996) und werden deshalb als relativ veränderungsresistent angesehen (Pajares 1992). Allerdings wird auch der akademischen Lehrkräftebildung und -fortbildung, insbesondere aber den Erfahrungen im Unterrichten in Verbindung mit einer kritischen Reflexion dieser Unterrichtspraxis, das Potenzial für die Beeinflussung von bildungsbezogenen Überzeugungen zugesprochen (Biedermann et al. 2015; Pajares 1992; Richardson 1996). Selbstwirksamkeitserwartungen entstehen und entwickeln sich Bandura (1997) zufolge aufgrund von Informationen über die eigenen Fähigkeiten, die aus vier Quellen stammen: direkte Erfahrungen, stellvertretende Erfahrungen, verbale Überzeugung und physiologische/emotionale Reaktionen. Direkte Erfahrungen spielen im Allgemeinen bei der Entwicklung von Selbstwirksamkeitserwartungen die größte Rolle, jedoch können auch stellvertretende Erfahrungen ähnlich wirksam sein (Bandura 1997; PfitznerEden 2016). Die Beziehung zwischen (Selbst-) Überzeugungen und Handeln ist damit als reziprok anzusehen: „Beliefs are thought to drive actions; however, experiences and reflection on action may lead to changes in and/or additions to beliefs" (Richardson 1996, S. 104).

Lehrkräfte(fort-)bildung und Unterrichtspraxis - Orte, an denen auch direkte, indirekte, physiologische/emotionale und Feedback-Erfahrungen mit den eigenen Fähigkeiten gemacht werden - bezeichnen wir unter Rückgriff auf das AngebotsNutzungsmodell von Helmke (2009), das von Kunter et al. (2011) auf die Entwicklung professioneller Kompetenz von Lehrkräften übertragen wurde und in der Lern-, Unterrichts- und Lehrkräfteforschung im deutschsprachigen Raum weit verbreitet ist, als Lerngelegenheiten. Neben persönlichen Voraussetzungen der Lehrkraft und dem schulischen Kontext können diesem Modell zufolge Lerngelegenheiten und deren Nutzung als die zentralen Faktoren der Kompetenzentwicklung von Lehrkräften angesehen werden (s. Abb. 1).

Vor diesem Hintergrund gehen wir grundsätzlich davon aus, dass sich die Nutzung von Lerngelegenheiten zu inklusivem Unterricht positiv auf die Entwicklung der professionellen Kompetenz von Lehrkräften und damit auch auf deren Überzeugungen und Selbstwirksamkeitserwartungen zu inklusivem Unterricht auswirkt.

Wie erwähnt gehören zu den relevanten Lerngelegenheiten Erfahrungen im Unterrichten von Schülerinnen und Schülern (direkte und physiologische/emotionale Erfahrungen) oder Hospitationen in inklusiven Settings (stellvertretende Erfahrungen 


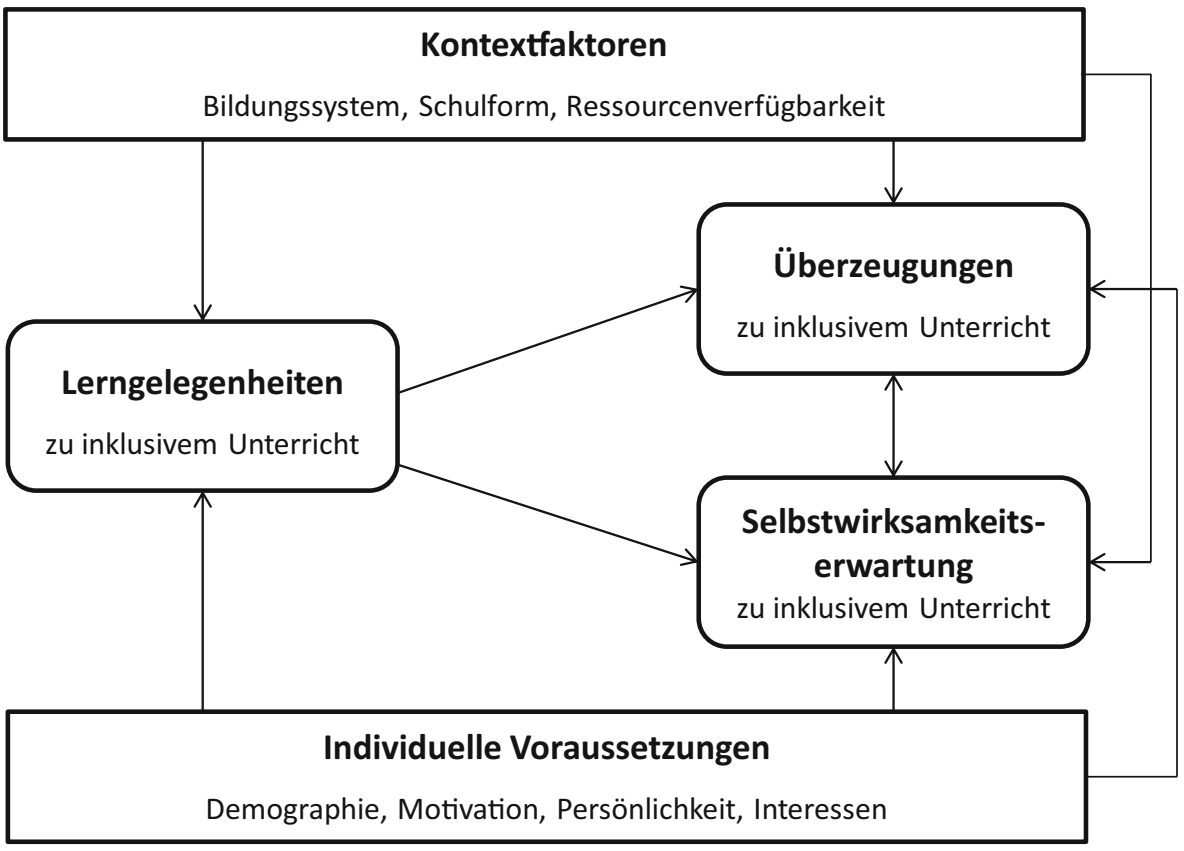

Abb. 1 Der Einfluss von Lerngelegenheiten auf (Selbst-)Überzeugungen. (Quelle: eigene Darstellung)

durch die Beobachtung und das Kennenlernen von Handlungsmodellen), inklusionsspezifische Aus- und Weiterbildungen oder inklusionsbezogene Veranstaltungen der Lehrkräftebildung (stellvertretende Erfahrungen). Im Einklang mit dem postulierten Kausalmodell ergaben bisherige empirische Untersuchungen, dass der Besuch spezifischer Weiterbildungen mit einer positiveren Einstellung gegenüber Inklusion einhergeht (Kim 2011), dass sich Erfahrungen im inklusiven Unterrichten positiv auf die Überzeugungen zur Inklusion auswirken (de Boer et al. 2011; Eichfeld und Algermissen 2016; Hellmich und Görel 2014) und zu höheren inklusionsbezogenen Selbstwirksamkeitserwartungen führen (Hellmich und Görel 2014; Malinen et al. 2013; Miesera und Gebhardt 2018; Schwab et al. 2017) und dass spezifische Lehrveranstaltungen die Selbstwirksamkeitserwartung zum Umgang mit Heterogenität (nicht aber entsprechende Einstellungen) fördern (Junker et al. 2020). Ausschließliche Berufserfahrung im segregierten Schulsystem korreliert dagegen eher negativ mit den Überzeugungen zur Inklusion (Eichfeld und Algermissen 2016; Gebhardt et al. 2015). Civitillo et al. (2016) betonten darüber hinaus die besondere Bedeutung der Lehrkräftebildung als einer geeigneten Plattform zur Unterstützung von angehenden Lehrkräften bei der Veränderung negativer inklusiver Überzeugungen.

Im Einklang mit den theoretischen Überlegungen und bisherigen empirischen Ergebnissen erwarten auch wir, dass Erfahrungen in Form von Lerngelegenheiten zu inklusivem Unterricht sich auf die Überzeugungen und Selbstwirksamkeitserwartungen von Lehrkräften auswirken: 
H1 Je mehr Lerngelegenheiten zu inklusivem Unterricht (angehende) Lehrkräfte nutzen konnten, desto positiver sind ihre Überzeugungen und Selbstwirksamkeitserwartungen zum inklusiven Unterricht.

Hinsichtlich des Kontextes für Lerngelegenheiten, Überzeugungen und Selbstwirksamkeitserwartungen (s. Abb. 1) spielt der angestrebte bzw. erreichte Lehramtsabschluss eine wichtige Rolle. So wird im Studium für angehende Lehrkräfte an Gymnasien und Gesamtschulen ein stärkerer Schwerpunkt auf die Fachausbildung gelegt, während im Grund-, Haupt- und Realschullehramt eher Fachdidaktik und Erziehungswissenschaft im Fokus stehen (Hascher 2014). Und auch wenn inzwischen in den meisten Ländern die Regelungen für das Lehramtsstudium so angepasst wurden, dass inklusionsbezogene Themen verpflichtender Studienbestandteil sind (Lange 2017), so geschah dies zum Teil nicht oder nicht in demselben Umfang für alle Lehramtstypen. So wie inklusiver Unterricht in unterschiedlichem Maße in den verschiedenen Schulformen implementiert ist - häufiger in Grund-, Hauptund Gesamtschulen, seltener in Gymnasien und beruflichen Schulen (Autorengruppe Bildungsberichterstattung 2018; Miesera und Gebhardt 2018) -, so variieren auch Lerngelegenheiten zur Inklusion je nach Art der Lehramtsausbildung.

Die Befundlage zum Zusammenhang von Schulform/Lehramtstyp und (Selbst-) Überzeugungen ist nicht eindeutig (Ruberg und Porsch 2017). In Übereinstimmung mit dem Argument quantitativ unterschiedlicher Lerngelegenheiten ergab aber die Studie von Langner (2015), dass Grundschullehrkräfte die höchste Bereitschaft zu inklusivem Unterricht aufweisen, Lehrkräfte an Gymnasien die geringste. Specht et al. (2016) stellten darüber hinaus für Kanada fest, dass angehende Lehrkräfte der Vorschul- und Primarstufe gegenüber Lehramtsstudierenden, die höhere Klassen unterrichten wollen, eine höhere Selbstwirksamkeitserwartung in Bezug auf das gemeinsame Unterrichten von Schülerinnen und Schülern mit und ohne SPF zeigen. Während der Umgang mit Inklusion das Kerngeschäft der Sonder-/Förderschullehrkräfte ist, betrifft er nur einen Teilbereich der unterrichtlichen Tätigkeit von Regelschullehrkräften. Infolgedessen fürchten insbesondere Lehrkräfte an Regelschulen oftmals, Schülerinnen und Schülern mit SPF nicht gerecht werden zu können (Gebhardt et al. 2018). Die Befunde von Miesera (2018) weisen in eine ähnliche Richtung wie diejenigen der zuvor genannten Studien. Vor diesem Hintergrund formulieren wir die folgende Hypothese:

H2 (Angehende) Sonder-/Förderschullehrkräfte verfügen im Lehramtsvergleich über die höchsten, (angehende) Gymnasiallehrkräfte hingegen über die niedrigsten Überzeugungen und Selbstwirksamkeitserwartungen zum inklusiven Unterricht.

Für die Nutzung von Lerngelegenheiten und die Möglichkeit, berufliche Erfahrungen zu sammeln, ist darüber hinaus relevant, in welcher Phase der Lehramtsausbildung bzw. Berufstätigkeit sich die (angehende) Lehrkraft befindet. So eignen sich Lehramtsstudierende mit fortschreitendem Studium mehr stellvertretende Erfahrungen in Form von Seminaren und Fachkursen und teilweise auch direkte Erfahrungen in Schulpraktika an, während im Referendariat und der aktiven Tätigkeit als Lehrkraft eigene Unterrichtserfahrungen an Bedeutung gewinnen. Entsprechend der unterschiedlichen Zahl und Qualität der Lerngelegenheiten sollten sich je nach 
der ausbildungs- und berufsbiografischen Phase die Überzeugungen und Selbstwirksamkeitserwartungen von (angehenden) Lehrkräften unterscheiden.

Es gibt einige, nicht immer konsistente Befunde zur Entwicklung von Überzeugungen und Selbstwirksamkeitserwartungen während des Lehramtsstudiums (im Hinblick auf Überzeugungen zur Inklusion: Bosse und Spörer 2014; Greiner et al. 2020; Hecht und Weber 2020; Hecht et al. 2016; Kraska und Boyle 2014; im Hinblick auf Selbstwirksamkeitserwartungen: Greiner et al. 2020; Hecht und Weber 2020; Pfitzner-Eden 2016). Beim Übergang in den Beruf, der angesichts der in unserer Studie benutzten Daten (s. Abschn. 3.1) von größerem Interesse ist, wurde in verschiedenen Studien in unterschiedlichen Ländern ein zumindest vorübergehender Rückgang positiver bzw. fortschrittlicher Einstellungen bei Lehrkräften beobachtet. So stellten Müller-Fohrbrodt et al. (1978) in einer Längsschnittstudie fest, dass in den ersten beiden Berufsjahren innovative, reformorientierte pädagogische Einstellungen von konservativen Einstellungen abgelöst werden, um in den nachfolgenden Berufsjahren wieder auf das Niveau am Ende des Studiums anzusteigen (,Konstanzer Wanne“; vgl. dazu Messner und Reusser 2000; Zingg und Grob 2002). Erklärt werden die Veränderungen beim Berufseinstieg durch die hohen, zu Überforderung führenden Belastungen (Zingg und Grob 2002) oder durch Diskrepanzerfahrungen zwischen ,idealistic notions developed during teacher education programs [...] and pressure from schools to rely on traditional patterns of behavior" (Brouwer und Korthagen 2005, S. 155). Auch in Bezug auf inklusiven Unterricht wurde ein Rückgang der positiven Einstellungen zwischen der Zeit kurz vor dem Studienabschluss und nach dem Berufseinstieg gefunden (Hecht et al. 2016; Hecht und Weber 2020). Die Einstellungen scheinen sich aber nicht im Sinne der „Konstanzer Wanne“ im weiteren Verlauf der Berufstätigkeit zurück auf das Ausgangsniveau bei Studienabschluss zu entwickeln. So stellten Boyle et al. (2013) in ihrer Studie mit Lehrkräften an schottischen Sekundarschulen fest, dass Lehrkräfte unmittelbar bei Berufseinstieg deutlich positivere Überzeugungen zu inklusiver Bildung aufweisen als Lehrerinnen und Lehrer mit längerer Berufserfahrung (ähnlich auch die Ergebnisse von Sermier Dessemontet et al. (2011) für die französischsprachige Schweiz).

Auch die bisherige, nicht sehr umfangreiche Forschung zur Entwicklung der Lehrerselbstwirksamkeitserwartung beim Berufseinstieg spricht für einen (zeitweiligen) Rückgang. So kam die Längsschnittstudie von Swan et al. (2011) mit einem vom Studium bis in die Berufstätigkeit reichenden Beobachtungsfenster und einer kleinen Kohorte von US-amerikanischen Lehramtsstudierenden zu dem Ergebnis: ,,...] individuals reported the lowest levels of teacher self-efficacy at the conclusion of their first year of teaching" (Swan et al. 2011, S. 136). Auch Woolfolk Hoy und Burke Spero (2005), die in einer US-amerikanischen Panelstudie verschiedene Selbstwirksamkeitsmaße bei (angehenden) Lehrkräften am Ende des Studienprogramms sowie des ersten Jahres der Berufstätigkeit untersuchten, ermittelten eine rückläufige Entwicklung und erklärten sie damit, dass Lehrkräfte zu Beginn ihrer Berufslaufbahn häufig die Komplexität ihrer Aufgaben unterschätzen und ihre optimistische Selbsteinschätzung in der Konfrontation mit der komplexen Realität getrübt wird. Und für Deutschland beobachteten Richter et al. (2013), dass die Selbstwirksamkeitserwartung von angehenden Mathematiklehrkräften im ersten Jahr des Referendariats signifikant zurückging. 
Entgegen der generellen Annahme eines positiven Zusammenhangs zwischen Lerngelegenheiten und Überzeugungen/Selbstwirksamkeitserwartungen zu inklusivem Unterricht erwarten wir daher, dass mit Fortschreiten der Lehramtsausbildung und im Berufsverlauf (angehende) Lehrkräfte zwar mehr Lerngelegenheiten nutzen können und dadurch auch positivere Überzeugungen und Selbstwirksamkeitserwartungen zur Inklusion entwickeln, jedoch die Übergänge von der eher theoretischen ersten bzw. zweiten Phase der Lehramtsausbildung zur zweiten bzw. dritten Phase, d.h. zur Berufspraxis, mit Brüchen in der Wahrnehmung der eigenen Kompetenzen und in den Überzeugungen zur Umsetzbarkeit und Qualität inklusiven Unterrichts einhergehen. Unsere Hypothesen zu diesen gegenläufigen Entwicklungen lauten deshalb:

H3a Je weiter fortgeschritten innerhalb der Lehramtsausbildung eine (angehende) Lehrkraft ist, desto mehr Lerngelegenheiten konnte sie nutzen.

H3b Im Vergleich zu Lehramtsstudierenden verfügen Referendarinnen und Referendare über negativere, im Vergleich zu erwerbstätigen Lehrkräften zu Beginn ihrer beruflichen Laufbahn hingegen über positivere Überzeugungen und Selbstwirksamkeitserwartungen zum inklusiven Unterricht.

\section{Daten und Methode}

\subsection{Stichprobe}

Die Untersuchung basiert auf Daten des Lehramtsstudierendenpanels (LAP), einer Zusatzstudie zur Startkohorte „Studierende“ des Nationalen Bildungspanels (NEPS) (https://doi.org/10.5157/NEPS:SC5:11.0.0; Blossfeld et al. 2011). Analysiert wird die überproportional gezogene Zufallsstichprobe von Studienanfängerinnen und Studienanfängern des Wintersemesters 2010/2011 in Lehramtsstudiengängen, die das gesamte Bundesgebiet sowie alle Arten von Lehramtsstudiengängen abdeckt. Die Variablen zu den Lerngelegenheiten, Überzeugungen und Selbstwirksamkeitserwartungen in Bezug auf inklusiven Unterricht wurden in einer Onlinebefragung im Herbst 2016 erhoben; weitere relevante Variablen wurden in früheren Panelwellen erfasst. Ausgewählte Kennwerte des Analysesamples, das Personen mit Lehramtsbezug und gültigen Angaben zu den inklusionsbezogenen Kompetenzen umfasst, sind in Tab. 1 wiedergegeben.

\subsection{Operationalisierung}

Eine Übersicht zur Operationalisierung der zentralen Variablen und Konstrukte bietet Tab. 2, die vollständigen Instrumente sind im Online-Anhang in Tab. A1 dargestellt. 
Tab. 1 Zusammensetzung des Analysesamples. (Quelle: NEPS/LAP)

\begin{tabular}{ll}
\hline Merkmal & Anteil/Mittelwert \\
\hline Geschlecht & $23 \%$ \\
Männlich & $77 \%$ \\
Weiblich & \\
Lehramtsstatus $^{a}$ & $45 \%$ \\
Studium & $41 \%$ \\
Referendariat $_{\text {Erwerbstätigkeit }}$ & $14 \%$ \\
Lehramtstyp $^{\text {Grundschule }}$ & \\
Sekundarstufe I $^{\mathrm{b}}$ & $15 \%$ \\
Gymnasium $^{\mathrm{c}}$ & $18 \%$ \\
Sonder-/Förderschule $^{\text {Berufliche Schule }}{ }^{\mathrm{d}}$ & $54 \%$ \\
Alter (Mittelwert in Jahren) $_{N}$ & $8 \%$ \\
\hline
\end{tabular}

a 168 Personen, die ihr Studium oder Referendariat bereits abgeschlossen haben, jedoch noch nicht in den jeweils nachfolgenden Status übergegangen sind, wurden der jeweils früheren Statusgruppe zugeordnet

${ }^{\mathrm{b}}$ Real-, Haupt- und Gesamtschulen

${ }^{c}$ Einschließlich Sekundarstufe II an allgemeinbildenden Schulen

${ }^{\mathrm{d}}$ Einschließlich beruflicher Gymnasien

Tab. 2 Erhebungsinstrumente. (Quelle: NEPS/LAP)

\begin{tabular}{|c|c|c|c|}
\hline $\begin{array}{l}\text { Variablen und Kon- } \\
\text { strukte }\end{array}$ & $\begin{array}{l}\text { Anzahl } \\
\text { Items }\end{array}$ & Beispielitem & $\begin{array}{l}\text { Cronbach } \\
\text { Alpha }\end{array}$ \\
\hline $\begin{array}{l}\text { Lerngelegenheiten } \\
\text { (gesamte Skala) }^{\mathrm{a}}\end{array}$ & 4 & $\begin{array}{l}\text { Wie oft hatten Sie (bisher) in Ihrer Lehrerausbildung } \\
\text { Gelegenheit, die folgenden Dinge zu lernen? } \\
\text { Den Bedürfnissen von Schülerinnen und Schülern mit } \\
\text { geistiger Behinderung gerecht werden. }\end{array}$ & 0,85 \\
\hline $\begin{array}{l}\text { Berufliche Erfahrung } \\
\text { (Inklusion) }^{\mathrm{b}}\end{array}$ & 1 & $\begin{array}{l}\text { Haben Sie Erfahrung im Unterrichten von Schülerin- } \\
\text { nen und Schülern mit besonderem Förderbedarf? }\end{array}$ & - \\
\hline $\begin{array}{l}\text { Überzeugungen zur } \\
\text { Gestaltung inklusi- } \\
\text { ven Unterrichts }{ }^{c}\end{array}$ & 3 & $\begin{array}{l}\text { Ein gemeinsamer Unterricht von Kindern und Ju- } \\
\text { gendlichen mit und ohne Behinderungen kann durch } \\
\text { entsprechende Methoden allen Kindern und Jugendli- } \\
\text { chen gerecht werden. }\end{array}$ & 0,80 \\
\hline $\begin{array}{l}\text { Überzeugungen } \mathrm{zu} \\
\text { den Effekten inklusi- } \\
\text { ven Unterrichts }\end{array}$ & 3 & $\begin{array}{l}\text { Schülerinnen und Schüler mit Behinderungen haben } \\
\text { höhere Lernzuwächse, wenn sie in Regelklassen } \\
\text { unterrichtet werden. }\end{array}$ & 0,72 \\
\hline $\begin{array}{l}\text { Selbstwirksamkeits- } \\
\text { erwartung in Be- } \\
\text { zug auf inklusiven } \\
\text { Unterricht }^{\mathrm{d}}\end{array}$ & 4 & $\begin{array}{l}\text { Ich kann Unterricht so gestalten, dass auch Schü- } \\
\text { lerinnen und Schüler mit Lernbeeinträchtigungen } \\
\text { erfolgreich am Unterricht teilhaben können. }\end{array}$ & 0,86 \\
\hline \multicolumn{4}{|c|}{ 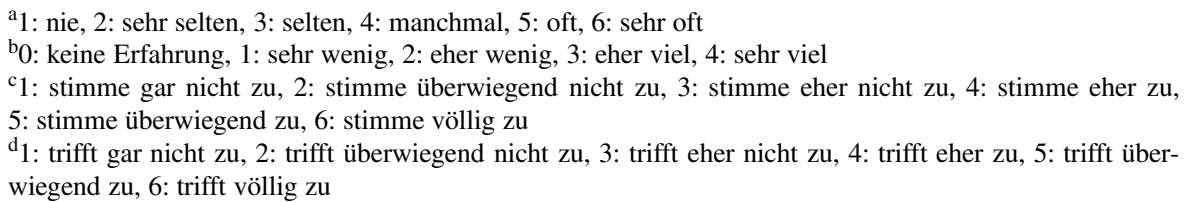 } \\
\hline
\end{tabular}




\subsection{1 Überzeugungen und Selbstwirksamkeitserwartungen in Bezug auf inklusiven Unterricht}

Für die Überzeugungen und Selbstwirksamkeitserwartung in Bezug auf inklusiven Unterricht wurden adaptierte und gekürzte Versionen eines Instruments von Bosse und Spörer (2014) verwendet. Ein Teil des Instruments bezieht sich laut den Autorinnen auf Einstellungen zum inklusiven Unterricht, erfasst nach unserem in Abschn. 2.1 dargelegten Begriffsverständnis jedoch eher inklusionsbezogene Überzeugungen.

Diese wurden mit zwei Subdimensionen zu je drei Items erhoben: Die Skala Überzeugungen zur Gestaltung inklusiven Unterrichts $(G)$ erfasst die Einschätzung zur generellen Umsetzbarkeit von Inklusion und zur Qualität inklusiven Unterrichts; die Skala Überzeugungen zu den Effekten inklusiven Unterrichts $(E)$ fokussiert die Auswirkungen inklusiver Lernsettings auf Schülerinnen und Schüler mit und ohne SPF. Beide Subskalen weisen zufriedenstellende interne Konsistenzen auf $(\alpha=0,80$ bzw. $\alpha=0,72 ;$ s. Tab. 2). In die nachfolgenden Analysen wurden die beiden Überzeugungsdimensionen als zweifaktorielles Modell unter Berücksichtigung einer Residualkorrelation $(\mathrm{r}=0,40)$ zwischen den negativ formulierten Items G2 und G3 (vollständiges Instrument im Online-Anhang in Tab. A1) einbezogen. Das Messmodell zeigte eine sehr hohe latente Korrelation zwischen den Faktoren ( $r=0,97)$, erwies sich jedoch im Vergleich mit Alternativmodellen (s. Tab. A2 im Online-Anhang) als am besten und insgesamt sehr gut an die Daten angepasst (RMSEA $=0,041$; $\mathrm{CFI}=0,995$; TLI $=0,989$; SRMR $=0,015$; zu akzeptablen Cut-off-Werten vgl. Arzheimer (2016) und Hu und Bentler (1999)). Für das Zweifaktorenmodell spricht auch - wie die nachfolgenden Analysen zeigen - die unterschiedliche Vorhersagekraft der Lerngelegenheiten und beruflichen Erfahrungen.

Die Selbstwirksamkeitserwartung (S) wurde anhand von vier Items erfasst. Für die nachfolgenden Analysen wurde eine Residualkorrelation zwischen den beiden Items S3 und S4 zugelassen $(r=0,31)$. Diese Fehlerkorrelation ist inhaltlich plausibel, da es in den betroffenen Items um Differenzen hinsichtlich Leistung bzw. Lernerfolg geht, während die beiden anderen Items explizit Schülerinnen und Schüler mit geistiger Entwicklungsverzögerung thematisieren. Die Faktorladungen liegen zwischen $\lambda=0,56$ und $\lambda=0,89$, der Modellfit (RMSEA $=0,030$; CFI = 1,000; TLI $=0,997$; SRMR $=0,004)$ und die interne Konsistenz $(\alpha=0,86)$ sind sehr gut.

\subsubsection{Inklusionsbezogene Lerngelegenheiten und Erfahrungen}

Die beruflichen Erfahrungen (B) im Unterrichten von Schülerinnen und Schülern mit besonderem Förderbedarf wurden anhand eines geringfügig umformulierten Items aus dem Projekt E1NS der Universität Hildesheim (2016; vgl. Schmieder 2019) erfasst, die in der Lehrkräfteausbildung gebotenen Lerngelegenheiten (L) mit einem geringfügig modifizierten und gekürzten Instrument aus der TEDS-M Studie (Laschke und König 2014). Die vier Items zu den Lerngelegenheiten $(\alpha=0,85$; s. Tab. 2) wurden aufgrund ihres Fokus auf unterschiedliche Förderbedarfe (motorische/geistige Entwicklung vs. Verhaltensauffälligkeiten/Lernschwierigkeiten) auf zwei Faktoren aufgeteilt. Den Faktor, der sich auf Schülerinnen und Schüler 
mit einem motorischen oder geistigen Förderbedarf bezieht, bezeichnen wir als „Lerngelegenheiten: motorische/geistige Entwicklung“. „Lerngelegenheiten: soziale/emotionale Entwicklung" haben wir den Faktor genannt, bei dem es um den Unterricht von Schülerinnen und Schülern mit emotionalen Problemen, Verhaltensauffälligkeiten oder Lernschwierigkeiten geht. Dieses Zwei-Faktoren-Modell erwies sich im Modellvergleich auf Basis der geprüften Fitindizes und des $\chi^{2}$-Differenztests als am besten an die Daten angepasst (RMSEA =0,050; CFI =0,999; TLI =0,993; $\mathrm{SRMR}=0,004)$.

\subsubsection{Kontextmerkmale der Lehrkräfteausbildung und Individualmerkmale}

Als weitere Erklärungsfaktoren für inklusionsbezogene Kompetenzen wurden zwei Kontextmerkmale berücksichtigt. Zum einen ging der Stand der Lehramtsausbildung bzw. Erwerbstätigkeit als Lehrkraft („Lehramtsstatus“) als Dummyvariablen in die Analysen ein. Zum anderen wurde der (angestrebte) Lehramtstyp berücksichtigt und ebenfalls in Form von Dummyvariablen analysiert. Wegen möglicher Zusammenhänge mit inklusionsbezogenen Überzeugungen und Selbstwirksamkeitserwartungen wurde aus dem Spektrum individueller Merkmale das Geschlecht der (angehenden) Lehrkräfte als Kontrollvariable einbezogen. Zwar sind die diesbezüglichen bisherigen Befunde nicht einheitlich, aber in mehreren Studien konnte ein Einfluss des Geschlechts zumindest auf Einstellungen zur Inklusion gefunden werden, wobei Frauen zumeist positivere Einstellungen als Männer aufweisen (de Boer et al. 2011 (Meta-Analyse); Ahmmed et al. 2012 (umgekehrter Zusammenhang); Boyle et al. 2013; Junker et al. 2020; Scheer et al. 2015).

\subsection{Methodisches Vorgehen}

Der erste Analyseschritt dieser Untersuchung, dessen Ergebnisse bereits in Abschn. 3.2 berichtet wurden, bestand in der Überprüfung der Faktorenstruktur aller relevanten Konstrukte mithilfe konfirmatorischer Faktorenanalysen. Konkurrierende Modelle mit einer unterschiedlichen Anzahl latenter Faktoren wurden anhand ausgewählter Fitindizes sowie $\chi^{2}$-Differenztests (Likelihood Ratio-Tests) verglichen (Christ und Schlüter 2012). Im Online-Anhang sind die Fitstatistiken aller geprüften Modelle (Tab. A2) sowie Informationen zu den Messmodellen (Abb. A1-A3) aufgeführt. In einem zweiten Schritt wurden die Mittelwerte der zentralen abhängigen und unabhängigen Merkmale ermittelt und auf signifikante Unterschiede zwischen ausgewählten Teilgruppen untersucht. Schließlich wurden Strukturgleichungsmodelle zur Überprüfung der aufgestellten Hypothesen geschätzt.

Um die Differenzen zwischen den verschiedenen Ausprägungen der polytomen kategorialen Merkmale Lehramtsstatus und Lehramtstyp auf Signifikanz zu testen, wurden die Strukturgleichungsmodelle mit unterschiedlichen Referenzkategorien analysiert. In Tab. 5 werden lediglich die Koeffizienten für das Modell mit den Referenzkategorien „Gymnasium“ für den Lehramtstyp und „Studium“ für den Lehramtsstatus wiedergegeben. Die Koeffizienten für andere Referenzkategorien ergeben sich als Differenz zwischen den aufgeführten Koeffizienten der entsprechenden Kategorien, wobei Rundungsfehler in Höhe von maximal 0,01 auftreten können. Ob 
sie mit einer Irrtumswahrscheinlichkeit von 5\% signifikant sind, wird durch den Buchstaben der Vergleichskategorie symbolisiert. Darüber hinaus wurden die indirekten und Gesamteffekte ermittelt und Modelle für bestimmte Sets von erklärenden Variablen (zum einen Lerngelegenheiten und Erfahrungen; zum anderen Schulform, Lehramtsstatus, Geschlecht) geschätzt, um den allein auf diese Variablenblöcke zurückzuführenden Beitrag zur Varianzaufklärung der Zielvariablen Überzeugungen und Selbstwirksamkeitserwartung zu bestimmen. Die Ergebnisse der letztgenannten Analysen werden nur auszugsweise berichtet und sind nicht tabellarisch dargestellt.

Die Analysen wurden mit Stata 15.0 und Mplus 7.11 durchgeführt. Zur adäquaten Behandlung fehlender Werte auf den abhängigen Variablen wurde das FullInformation-Maximum-Likelihood-Schätzverfahren (FIML) verwendet.

\section{Ergebnisse}

\subsection{Deskriptive Analysen}

Die in Tab. 3 abgebildeten Mittelwerte lassen erkennen, dass sich die Befragten insgesamt betrachtet eher wenig Erfahrung $(M=1,82)$ im Unterrichten von Schülerinnen und Schülern mit SPF attestieren. Entsprechende Lerngelegenheiten haben sie im Rahmen von Lehramtsstudium und Referendariat nach eigener Einschätzung im Mittel selten (Unterrichten von Schülerinnen und Schülern mit Verhaltensauffälligkeiten und Lernschwierigkeiten: $M_{\mathrm{VL}}=2,93$ ) bzw. sehr selten (Unterrichten von Schülerinnen und Schülern mit motorischem oder geistigem Förderbedarf: $\left.M_{\mathrm{B}}=1,93\right)$ genutzt. Dennoch verfügen die (zukünftigen) Lehrkräfte über eine relativ hohe Selbstwirksamkeitserwartung $\left(M=3,91\right.$; ,trifft eher $\left.z u^{\text {") }}\right)$ sowie eine eher positive Überzeugung in Bezug auf inklusiven Unterricht (Gestaltung inklusiven

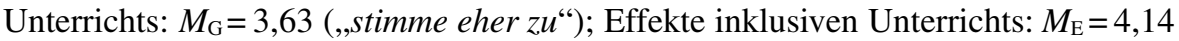

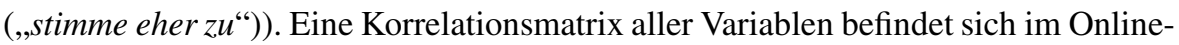
Anhang in Tab. A3.

In Tab. 4 sind die Mittelwerte differenziert nach Schulform, Lehramtsstatus und Geschlecht aufgeführt. Der zur Überprüfung von Hypothese H2 vorgenommene Schulformvergleich zeigt, dass (angehende) Sonder-/Förderschullehrkräfte über signifikant mehr Lerngelegenheiten und berufliche Erfahrungen im Unterrichten von

Tab. 3 Deskriptive Kennwerte. (Quelle: NEPS/LAP)

\begin{tabular}{lllllll}
\hline Konstrukt & Anzahl Items & $N$ & $M$ & SD & Min & Max \\
\hline $\begin{array}{l}\text { Lerngelegenheiten: soziale/emotionale } \\
\begin{array}{l}\text { Entwicklung } \\
\text { Lerngelegenheiten: motorische/geistige }\end{array}\end{array}$ & 2 & 1855 & 2,93 & 1,36 & 1 & 6 \\
Entwicklung & 2 & 1854 & 1,93 & 1,26 & 1 & 6 \\
Berufliche Erfahrung (Förderbedarf) & 1 & & & & & \\
Überzeugungen: Unterrichtsgestaltung & 3 & 1851 & 1,82 & 1,21 & 0 & 4 \\
Überzeugungen: Unterrichtseffekte & 3 & 1862 & 3,63 & 1,07 & 1 & 6 \\
Selbstwirksamkeitserwartung (Inklusion) & 4 & 1862 & 4,14 & 0,89 & 1 & 6 \\
\hline
\end{tabular}

${ }^{a}$ Umfasst Verhaltensauffälligkeiten und Lernschwierigkeiten 


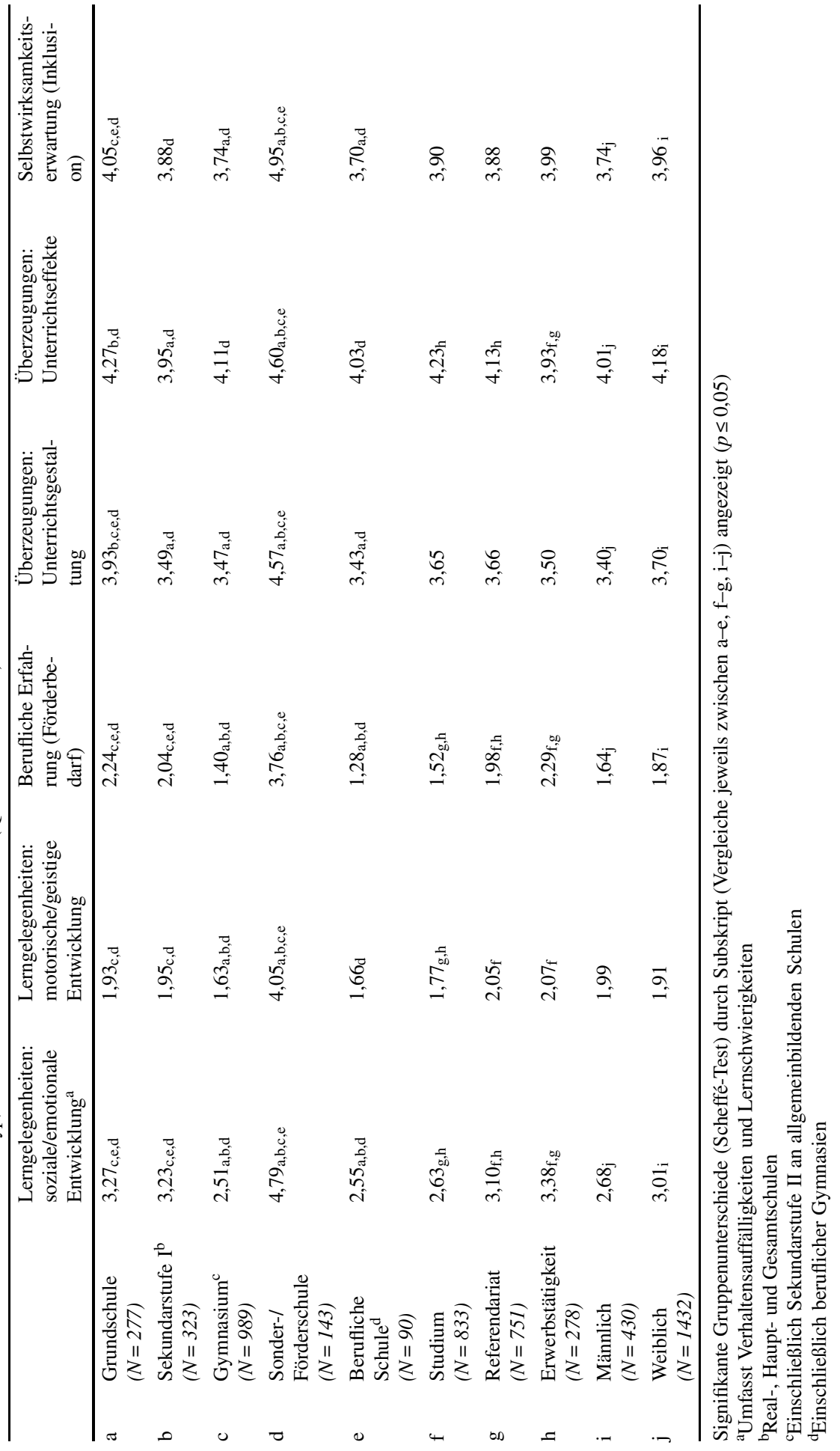


Schülerinnen und Schülern mit SPF verfügen als (angehende) Lehrkräfte aller anderen Schulformen. Die wenigsten Lerngelegenheiten und Erfahrungen berichteten (zukünftige) Lehrkräfte der Gymnasien und beruflichen Schulen. Die inklusionsbezogenen Überzeugungen und Selbstwirksamkeitserwartungen sind unter angehenden Lehrkräften der Sekundarstufe I, der Gymnasien und beruflichen Schulen ähnlich ausgeprägt und unterscheiden sich nicht signifikant voneinander. Etwas höher fallen die Mittelwerte der (angehenden) Grundschullehrkräfte aus. Die mit Abstand höchsten Werte bei den Überzeugungen und Selbstwirksamkeitserwartungen erzielen die (angehenden) Sonder-/Förderschullehrkräfte. Sie unterschieden sich damit statistisch bedeutsam von allen anderen Teilgruppen.

Mit Blick auf den in den Hypothesen $\mathrm{H} 3 \mathrm{a}$ und $\mathrm{H} 3 \mathrm{~b}$ thematisierten Lehramtsstatus zeigt sich im Großen und Ganzen, dass erwerbstätige Lehrkräfte gegenüber Referendarinnen und Referendaren und diese gegenüber Studierenden einen Vorsprung an Lerngelegenheiten und Erfahrungen aufweisen. In ihren Überzeugungen zur Gestaltung inklusiven Unterrichts sowie der Selbstwirksamkeitserwartung unterscheiden sich die drei Statusgruppen jedoch nicht signifikant voneinander. Lediglich bezüglich der Überzeugung zu den Effekten inklusiven Unterrichts weisen erwerbstätige Lehrkräfte signifikant niedrigere Werte auf als die Vergleichsgruppen.

Frauen verfügen im Vergleich zu Männern bei allen betrachteten Merkmalen mit Ausnahme der Lerngelegenheiten in Bezug auf Schülerinnen und Schüler mit motorischem und geistigem Förderbedarf über signifikant höhere Mittelwerte.

\subsection{Multivariate Analyse}

In Tab. 5 sind die Ergebnisse eines Strukturgleichungsmodells aufgeführt. Konstrukte mit mehr als einem Item wurden entsprechend den Voranalysen latent modelliert, die kategorialen Variablen dummykodiert. Die Ergebnisse der Schätzung zeigen zum einen, dass das spezifizierte Modell eine sehr gute Passung mit den empirischen Daten aufweist (RMSEA = 0,041; CFI=0,972; TLI=0,958; SRMR = 0,024). Sie zeigen entgegen der in Hypothese H1 formulierten Erwartung zum anderen, dass Lerngelegenheiten und Erfahrungen in der Lehramtsausbildung und den ersten Berufsjahren für die inklusionsbezogenen Überzeugungen von (angehenden) Lehrkräften keine große Relevanz haben. Während von den Lerngelegenheiten immerhin signifikante, wenn auch schwache Effekte auf die Überzeugung zur Gestaltung inklusiven Unterrichts ausgehen - wobei allein auf die Lerngelegenheiten elf Prozent der Varianz dieser Zielvariablen (bei 16\% Varianzaufklärung durch das Modells insgesamt) zurückgehen -, wird die Überzeugung zu den Effekten inklusiven Unterrichts, z. B. bezogen auf den Lernzuwachs von Schülerinnen und Schülern mit motorischem/geistigem Förderbedarf, von diesen Merkmalen gar nicht beeinflusst. Mit standardisierten Pfadkoeffizienten zwischen 0,10 und 0,29 deutlichere und hoch signifikante Effekte haben Lerngelegenheiten und Erfahrungen jedoch in Bezug auf die Selbstwirksamkeitserwartung. Bei einer Varianzaufklärung durch das Modell von insgesamt $39 \%$ erklären diese Kovariaten allein gut $36 \%$ der Varianz der inklusionsbezogenen Selbstwirksamkeitserwartung. Hinsichtlich der Überzeugung zu den Effekten inklusiven Unterrichts liegt die Varianzaufklärung durch das Modell insgesamt bei nur neun Prozent. 


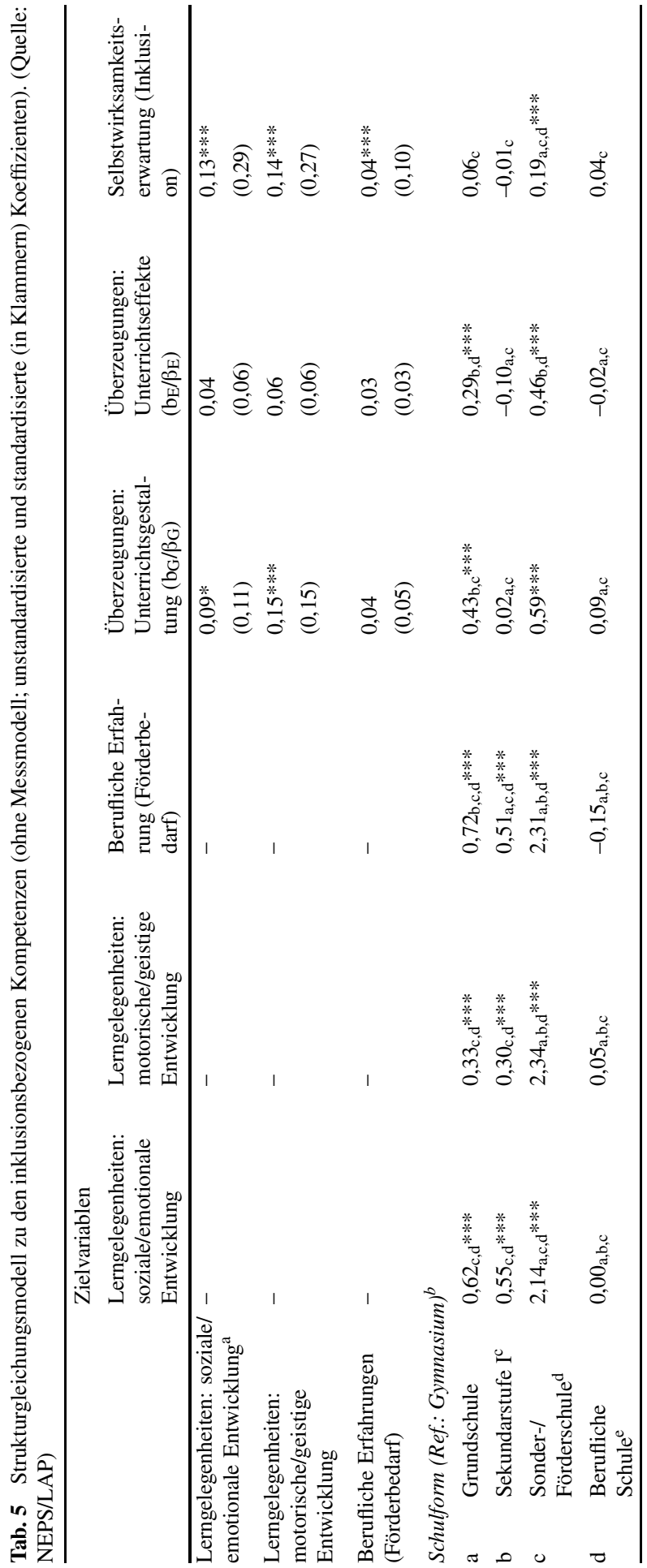




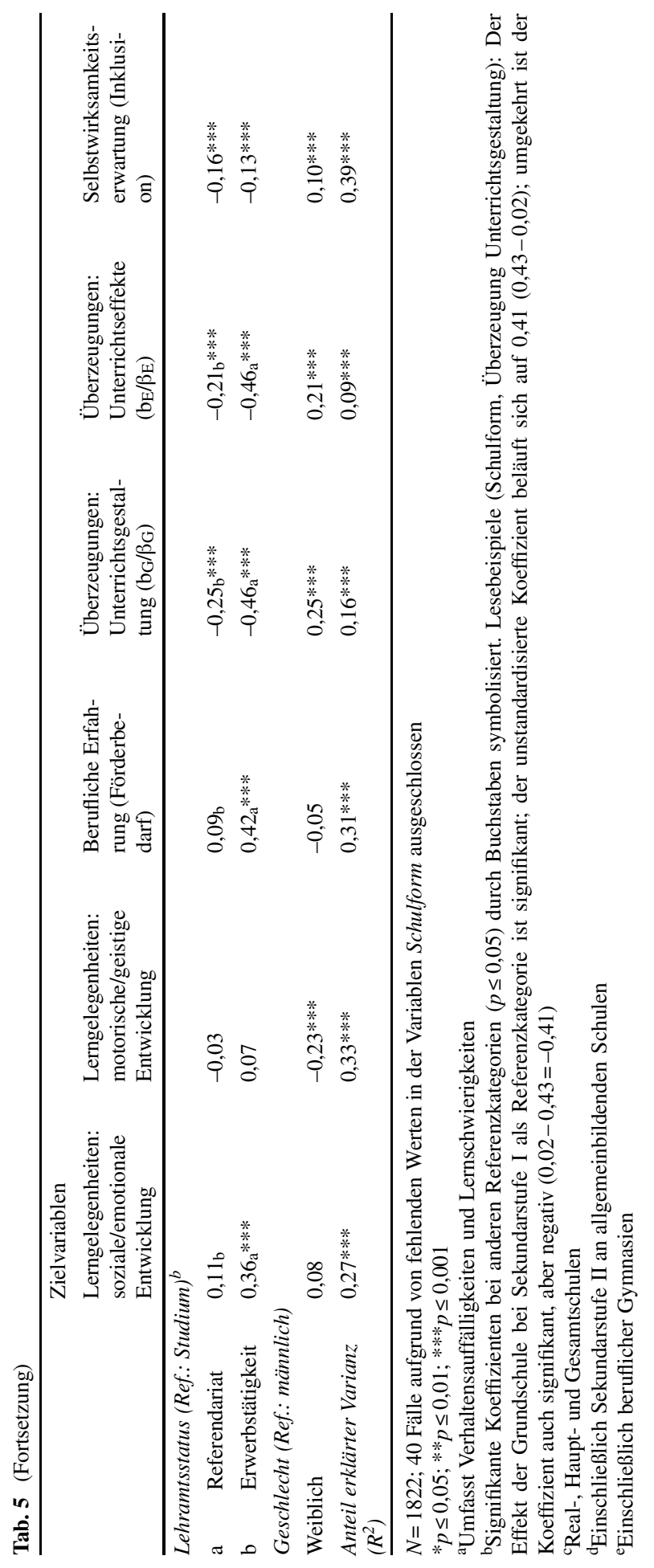


Wie in Hypothese $\mathrm{H} 2$ erwartet, berichteten (angehende) Lehrkräfte der Grundund insbesondere der Sonder-/Förderschulen im Vergleich zu jenen der Gymnasien (und auch der beruflichen Schulen) signifikant mehr Lerngelegenheiten und Erfahrungen im Unterrichten von Schülerinnen und Schülern mit besonderen Förderbedarfen; und sie stehen auch unabhängig davon der Inklusion positiver gegenüber als Lehrkräfte aller anderen Schulformen (unstandardisierter Effekt im Vergleich zum Gymnasium für Grundschule: $b_{G}=0,43$ und $b_{E}=0,29$; für Sonder-/Förderschule: $b_{G}=0,59$ und $\left.b_{E}=0,46\right)$. Ermittelt man den Gesamteffekt als Summe der direkten und der indirekten, über die Lerngelegenheiten und beruflichen Erfahrungen vermittelten Effekte, ergibt sich, dass sich Lehrkräfte an Sonder- und Förderschulen bei gleichem Lehramtsstatus und Geschlecht um mehr als einen Skalenpunkt positiver zum inklusiven Unterricht äußern als Lehrkräfte an Gymnasien $\left(\mathrm{b}_{\mathrm{G}(\text { gesamt }}=1,23\right)$ und beruflichen Schulen $\left(b_{\mathrm{G}(\text { gesamt })}=1,13\right)$.

Lehrkräfte der Sekundarstufe I haben in etwa gleichem Maße wie diejenigen der Grundschule Lerngelegenheiten genutzt und in etwas geringerem, allerdings auf dem Fünf-Prozent-Niveau signifikanten Maße einschlägige berufliche Erfahrungen machen können. Bei gleicher Ausprägung aller anderen analysierten erklärenden Variablen weisen sie aber - ähnlich wie die (angehenden) Lehrkräfte für Gymnasien und berufliche Schulen - deutlich negativere inklusionsbezogene Überzeugungen auf. In der Folge ist der Gesamteffekt des Lehramtstyps Sekundarstufe I gegenüber der Grundschule unabhängig von dem Lehramtsstatus signifikant negativ. Im Vergleich zu den (angehenden) Lehrkräften für Gymnasien und berufliche Schulen geben Befragte, die ein Lehramt der Sekundarstufe I anstreben oder ausüben, signifikant mehr inklusionsbezogene Lerngelegenheiten und berufliche Erfahrungen an. Der partielle Effekt in Bezug auf inklusionsbezogene Überzeugungen ebenso wie der entsprechende Gesamteffekt unterscheidet sich aber nicht signifikant zwischen diesen Lehrämtern.

Bei gleicher Berufserfahrung und gleicher Zahl an Lerngelegenheiten zeigen sich in Bezug auf die Selbstwirksamkeitserwartung kaum Effekte der Schulform. Nur (zukünftige) Sonder-/Förderschullehrkräfte heben sich von den anderen Befragten $a b$ : Sie verfügen gegenüber (angehenden) Lehrkräften an Gymnasien $(b=0,19)$, für die Sekundarstufe I $(b=0,20)$, berufliche Schulen $(b=0,15)$ und die Primarstufe $(b=0,13)$ über eine signifikant höhere Selbstwirksamkeitserwartung $(p \leq 0,05)$. Bei gleichem Stand der Lehramtsausbildung beläuft sich der Unterschied zwischen dem Sonder-/Förderschullehramt und den anderen Lehrämtern auf fast bis zu einem Skalenpunkt.

Mit Blick auf den in Hypothese H3a und H3b fokussierten Lehramtsstatus ist zu beobachten, dass erwerbstätige Lehrkräfte im Vergleich zu Studierenden und Befragten im Referendariat zwar signifikant mehr Lerngelegenheiten und Erfahrungen im Unterrichten von Schülerinnen und Schülern mit besonderem Förderbedarf berichten. In Bezug auf Lerngelegenheiten zum Unterrichten von Schülerinnen und Schülern mit motorischem/geistigem Förderbedarf ergeben sich aber keine signifikanten Effekte des Lehramtsstatus; auch sind - ceteris paribus - die Unterschiede in Lerngelegenheiten und beruflichen Erfahrungen zwischen Befragten im Studium und im Vorbereitungsdienst nicht signifikant. Unabhängig vom angestrebten oder ausgeübten Lehramt und Geschlecht weisen erwerbstätige Lehrkräfte im Vergleich zu Studie- 
renden aber ausgeprägt negativere inklusionsbezogene Überzeugungen $\left(b_{\mathrm{G}}=-0,51\right.$, $\left.\mathrm{b}_{\mathrm{E}}=-0,46 ; p \leq 0,001\right)$ und ein geringeres $\mathrm{Ma} \beta$ an Selbstwirksamkeitserwartungen $(\mathrm{b}=-0,13 ; p \leq 0,001)$ auf. Der Effekt des Vorbereitungsdienstes auf inklusionsbezogene Überzeugungen ist ebenfalls hoch signifikant und weist in die gleiche Richtung, ist aber weniger stark. Dabei sind auch die Unterschiede zwischen Befragten im Referendariat und erwerbstätigen Lehrkräften signifikant, so dass sich eine Rangfolge von Studierenden mit den positivsten Überzeugungen zum inklusiven Unterricht, über Referendarinnen und Referendaren mit signifikant weniger positiven Überzeugungen bis hin zu Erwerbstätigen mit den negativsten Überzeugungen ergibt. Eine zusätzlich vorgenommene Effektzerlegung kommt zu dem Ergebnis, dass der direkte negative Effekt der Erwerbstätigkeit auf die Überzeugungen so stark ist, dass er von dem indirekten positiven Effekt des größeren Umfangs an Lerngelegenheiten und beruflichen Erfahrungen nicht kompensiert werden kann und der Gesamteffekt hoch signifikant und negativ bleibt.

\section{Diskussion und Fazit}

\subsection{Zusammenfassende Diskussion}

Das Ziel der vorliegenden Untersuchung war die Erforschung von Effekten der inklusionsbezogenen Lerngelegenheiten und Unterrichtserfahrungen mit Schülerinnen und Schülern mit SPF auf die Ausprägung ausgewählter inklusionsbezogener Kompetenzfacetten. Die Ergebnisse der Analysen machten deutlich, dass sich die betrachteten erklärenden Merkmale positiv auf die Überzeugungen und Selbstwirksamkeitserwartungen von angehenden Lehrkräften sowie Berufsanfängerinnen und Berufsanfängern im Referendariat und im Schuldienst auswirken. Dieser Befund steht im Einklang mit der nationalen und internationalen integrations- und inklusionsbezogenen Lehrkräfteforschung (z.B. Avramidis und Norwich 2002; de Boer et al. 2011; Kopp 2009; Schwab et al. 2017).

Allerdings ist festzustellen, dass sich die inklusionsbezogenen Überzeugungen in deutlich geringem Ausmaß durch Lerngelegenheiten und Berufserfahrungen erklären lassen als die entsprechenden Selbstwirksamkeitserwartungen. Die Überzeugung zu den Effekten inklusiven Unterrichts wird von Lerngelegenheiten und beruflichen Erfahrungen sogar gar nicht beeinflusst. Insofern kann Hypothese H1 in Bezug auf die Überzeugungen nur eingeschränkt, in Bezug auf die Selbstwirksamkeitserwartung hingegen vollständig bestätigt werden. Die gefundene geringe Varianzaufklärung der inklusionsbezogenen Überzeugungen deckt sich mit dem empirischen Forschungsstand, der den (inklusionsbezogenen) Überzeugungen von (angehenden) Lehrkräften eine relativ hohe Stabilität im Zeitverlauf attestiert. Theoretisch erklären lässt sie sich durch die schon erwähnte Annahme, dass pädagogische Überzeugungen bereits in einem früheren Lebensabschnitt, etwa durch die eigene Schulzeit und frühe Erfahrungen im privaten Umfeld, geprägt und tief verankert werden und sich später nur eingeschränkt durch die Lehramtsausbildung beeinflussen lassen (Pajares 1992; Richardson 1996). Darüber hinaus wird davon ausgegangen, dass sich Überzeugungen nur sehr langsam als Ergebnis eines erlebten inklusionsbezogenen Kompetenzge- 
fühls verändern (Bosse et al. 2017; Hellmich und Görel 2014; Sermier Dessemontet et al. 2011).

Hypothese H2 zufolge sollten (angehende) Sonder-/Förderschullehrkräfte dem Unterrichten in inklusiven Schulklassen am positivsten und zuversichtlichsten gegenüberstehen, (zukünftige) Gymnasiallehrkräfte hingegen am kritischsten. Diese Annahme bestätigen schon die gruppenspezifischen Mittelwerte. Die multivariaten Analysen verdeutlichen, dass dieser Befund auch unter Kontrolle weiterer Merkmale wie Lerngelegenheiten und Erfahrungen, über die (angehende) Sonder-/ Förderschullehrkräfte deutlich häufiger verfügen als (angehende) Gymnasiallehrkräfte, Bestand hat. Dies ordnet sich nahtlos in den bestehenden Forschungsstand ein (Miesera 2018; Specht et al. 2016). Die besonders hohe inklusionsbezogene Kompetenz (angehender) Sonder-/Förderschullehrkräfte basiert neben der großen Zahl an Lerngelegenheiten und Erfahrungen auch darauf, dass sie sich mit ihrer Berufswahl bewusst für das Unterstützen und Fördern von Menschen mit sonderpädagogischen Förderbedarfen entschieden haben und zumeist bereits vor dem Studium Vorerfahrungen durch entsprechende Kontakte sammeln konnten (Gebhardt et al. 2018). Für (angehende) Lehrkräfte an Regelschulen ist das Thema Inklusion hingegen i.d.R. kein entscheidungsrelevantes Berufswahlkriterium, sondern vielmehr eine Thematik, mit der sie sich in ihrer Lehramtsausbildung erstmals dezidiert auseinandersetzen (Gebhardt et al. 2018).

Obwohl sich insgesamt ein positiver Zusammenhang zwischen Lerngelegenheiten und inklusionsbezogenen Kompetenzen abzeichnet, belegen die Analysen auch, dass erwerbstätige Lehrkräfte - wie in den Hypothesen H3a und H3b erwartet - trotz eines höheren Umfangs an Lerngelegenheiten negativere Überzeugungen und eine niedrigere Selbstwirksamkeitserwartung als Lehramtsstudierende aufweisen. Auch Befragte im Vorbereitungsdienst haben negativere Überzeugungen und Selbstwirksamkeitserwartungen als Lehramtsstudierende, verfügen aber über positivere Überzeugungen als erwerbstätige Lehrkräfte. Dies könnte auf einen doppelten Praxisschock beim Übergang in das Referendariat und die anschließende Erwerbstätigkeit hindeuten, der auch von Dicke et al. (2016) anhand der Entwicklung der emotionalen Erschöpfung beobachtet wurde. Die Klärung der Frage, wie sich inklusionsbezogene Überzeugungen und Selbstwirksamkeitserwartungen im weiteren Berufsverlauf entwickeln und ob sie sich im Sinne einer „Konstanzer Wanne“ erholen, ist zukünftiger Forschung vorbehalten. Die bisher dazu vorliegenden wenigen Studien haben, wie in Abschn. 2.2 dargelegt, keine eindeutige Befundlage ergeben.

\subsection{Limitationen}

Als eine Einschränkung dieser Studie ist zu nennen, dass die Messung der Lerngelegenheiten und Erfahrungen durch die subjektiv eingeschätzte Häufigkeit erfolgte und dabei die Qualität des Lernangebots und die Art der Erfahrungen nicht erhoben wurden. Wie erwähnt wirken Lerngelegenheiten sehr unterschiedlich je nachdem, ob sie direkte oder indirekte Erfahrungen vermitteln. Auch ist relevant, ob die Erfahrungen positiver oder negativer Natur sind. Positive Erfahrungen, insbesondere direkte positive Erfahrungen, fördern die Selbstwirksamkeitserwartung, während negative Erfahrungen das Gegenteil bewirken können (Bandura 1997). 
In Bezug auf die Messung der inklusionsbezogenen Selbstwirksamkeitserwartung ist zu konstatieren, dass die eingesetzten Items vornehmlich auf Schülerinnen und Schüler mit geistigen Entwicklungsverzögerungen und Lernbeeinträchtigungen Bezug nehmen, während andere Förderschwerpunkte wie körperliche Beeinträchtigungen keine explizite Erwähnung finden. Inwieweit dies unsere Ergebnisse beeinflusst, kann nicht abschließend beurteilt werden. Dafür, dass die Auswirkungen der eingeschränkten Messung der inklusionsbezogenen Selbstwirksamkeitserwartung eher gering sein dürften, sprechen die Befunde der Studie von Kopp (2009) zur Selbstwirksamkeitserwartung im Umgang mit besonderen Kindern. Ihre Faktorenanalyse von 25 Items (Benennung unterschiedlicher Förderbedarfe und Heterogenitätsdimensionen) zeigte, dass sich die Items nicht nach Merkmalen der Schülerinnen und Schüler gruppierten, sondern nach Art der Anforderungen an die Lehrkraft (z.B. Herstellung eines inklusiven Klassenklimas). Der Faktor , adaptive Unterrichtsgestaltung“, auf den auch der Fokus unserer Studie liegt, stellte sich als der konsistenteste, stärkste und bedeutsamste Faktor heraus.

Eine weitere Limitation dieser Studie besteht darin, dass Querschnittsanalysen durchgeführt wurden. Entsprechend kann nur approximativ über den Vergleich verschiedener Statusgruppen auf Entwicklungen geschlossen werden. Mit dem Vorliegen von Daten aus Wiederholungsmessungen wird es möglich sein, die Entwicklung der inklusionsbezogenen Kompetenzen im Längsschnitt zu betrachten und die „Konstanzer Wanne" bzw. die Auswirkung der Konfrontation mit der Unterrichtspraxis als intraindividuelle Entwicklung zu analysieren.

\subsection{Implikationen für die Forschung}

Entsprechend unserer Fragestellung basiert das Analysemodell unserer Untersuchung auf theoretischen Überlegungen, die einen kausalen Einfluss von bislang wahrgenommenen Lerngelegenheiten und gemachten Erfahrungen auf Überzeugungen und Selbstwirksamkeitserwartungen postulieren. Auch in der bisherigen empirischen Forschung zu dieser Thematik wird überwiegend ein derart gerichteter Zusammenhang angenommen (für Selbstwirksamkeitserwartung vgl. z. B. Bosse und Spörer 2014; Malinen et al. 2013; für Überzeugungen vgl. z. B. Greiner et al. 2020; Miesera und Gebhardt 2018). Theoretisch ist allerdings auch die umgekehrte Wirkungsrichtung, d.h. ein Einfluss von (Selbst-) Überzeugungen auf die zukünftige Wahrnehmung von Lerngelegenheiten, denkbar. Im Studium jedoch wird die Auswahl von Lerninhalten durch curriculare Vorgaben begrenzt. Zudem kann mit den in dieser Arbeit verwendeten Daten kein zeitverzögerter wechselseitiger Zusammenhang untersucht werden. Es erscheint jedoch lohnenswert, ihn im Rahmen zukünftiger längsschnittlicher Untersuchungen mit geeigneten Daten genauer, z. B. anhand von Cross-lagged-Panelmodellen, zu analysieren.

Auch hinsichtlich des kausalen Zusammenhangs zwischen inklusionsbezogenen Überzeugungen und Selbstwirksamkeitserwartungen besteht Forschungsbedarf. Während wir im vorliegenden Beitrag lediglich einen korrelativen Zusammenhang zwischen den abhängigen Variablen angenommen haben, wurden in anderen Studien gerichtete Zusammenhänge zwischen den beiden Konstrukten spezifiziert. Dabei wurde häufig von einem Effekt der Selbstwirksamkeitserwartung auf Überzeugun- 
gen bzw. Einstellungen ausgegangen und in verschiedenen Querschnittstudien auch bestätigt (z. B. Hellmich et al. 2016; Malinen et al. 2012; Sermier Dessemontet et al. 2011). Die Befunde von Längsschnittstudien waren auch hinsichtlich der Wirkrichtung weniger eindeutig. So ergab die Untersuchung von Hecht und Weber (2020) nicht, dass inklusionsbezogene Selbstwirksamkeitserwartungen zukünftige Einstellungen beeinflussen, sondern dass Selbstwirksamkeitserwartungen zum Teil auf bestimmte Einstellungen zurückzuführen sind. Savolainen et al. (2020) dagegen fanden in ihrer Cross-lagged-Panel-Analyse nur einen signifikanten positiven Einfluss der Selbstwirksamkeitserwartung auf Einstellungen zur Inklusion, aber keinen umgekehrten Effekt. Und Bosse et al. (2016) konnten in ihrem autoregressiven Modell zwar einen statistisch bedeutsamen Zusammenhang zwischen der Einstellung zur Gestaltung inklusiven Unterrichts und der entsprechenden Selbstwirksamkeitserwartung feststellen, jedoch keine kreuzverzögerten Effekte der inklusionsbezogenen Einstellungen und Selbstwirksamkeitserwartungen im Zeitverlauf beobachten.

In unserem Beitrag wurde der Einfluss von Lerngelegenheiten auf ausgewählte Aspekte der professionellen Kompetenz untersucht, die ihrerseits prädiktiv für professionelles Handeln sind. Eine folgerichtige und zukünftiger Forschung vorbehaltene Erweiterung unseres Analysemodells würde in der Aufnahme von inklusionsbezogenen Aspekten des beruflichen Handelns von Lehrkräften, z. B. hinsichtlich der Individualisierung und Differenzierung des Unterrichts, bestehen. Eine solche Studie könnte, vor allem wenn sie längsschnittlich angelegt ist, zur Absicherung des bislang nur auf wenigen Arbeiten beruhenden Kenntnisstands beitragen.

Die vorgestellte Untersuchung stärkt die vorliegende empirische Evidenz zu den Einflussfaktoren inklusionsbezogener Überzeugungen und Selbstwirksamkeitserwartungen und macht die bisherigen Ergebnisse aufgrund der schulform- und statusübergreifenden Analysen belastbarer. Gleichzeitig erweitert sie den vorhandenen Kenntnisstand, da differenzierte Ergebnisse für (angehende) Lehrkräfte verschiedener Schulform und in unterschiedlichen Phasen ihres Ausbildungs- und Berufsverlaufs vorgelegt werden konnten. Praktisch bedeutsam ist die Studie, da Lerngelegenheiten - anders als individuelle Merkmale wie Interessen oder psychosoziale Eigenschaften - leicht verändert werden können. Aus der theoretisch angenommenen und teilweise auch schon empirisch nachgewiesenen Relevanz von Überzeugungen und Selbstwirksamkeitserwartungen für die Unterrichtsgestaltung und darüber vermittelt für die Kompetenzen der Lernenden und aus dem vorgestellten Befund, dass Lerngelegenheiten in Lehramtsausbildung und Beruf über alle Schulformen hinweg $\mathrm{zu}$ positiveren inklusionsbezogenen Überzeugungen beitragen und die Selbstwirksamkeitserwartung erhöhen, kann gefolgert werden, dass Inklusion durch den qualitativen und quantitativen Ausbau von inklusionsbezogenen Lerngelegenheiten und Praxiserfahrungen gefördert wird.

Zusatzmaterial online Zusätzliche Informationen sind in der Online-Version dieses Artikels (https://doi. org/10.1007/s11618-021-01038-z) enthalten.

Funding Open Access funding enabled and organized by Projekt DEAL.

Open Access Dieser Artikel wird unter der Creative Commons Namensnennung 4.0 International Lizenz veröffentlicht, welche die Nutzung, Vervielfältigung, Bearbeitung, Verbreitung und Wiedergabe in jeglichem Medium und Format erlaubt, sofern Sie den/die ursprünglichen Autor(en) und die Quelle ord- 
nungsgemäß nennen, einen Link zur Creative Commons Lizenz beifügen und angeben, ob Änderungen vorgenommen wurden.

Die in diesem Artikel enthaltenen Bilder und sonstiges Drittmaterial unterliegen ebenfalls der genannten Creative Commons Lizenz, sofern sich aus der Abbildungslegende nichts anderes ergibt. Sofern das betreffende Material nicht unter der genannten Creative Commons Lizenz steht und die betreffende Handlung nicht nach gesetzlichen Vorschriften erlaubt ist, ist für die oben aufgeführten Weiterverwendungen des Materials die Einwilligung des jeweiligen Rechteinhabers einzuholen.

Weitere Details zur Lizenz entnehmen Sie bitte der Lizenzinformation auf http://creativecommons.org/ licenses/by/4.0/deed.de.

\section{Literatur}

Abele, A.E., \& Candova, A. (2007). Prädiktoren des Belastungserlebens im Lehrerberuf. Zeitschrift für Pädagogische Psychologie, 21(2), 107-118.

Ahmmed, M., Sharma, U., \& Deppeler, J. (2012). Variables affecting teachers' attitudes towards inclusive education in Bangladesh. Journal of Research in Special Educational Needs, 12(3), 132-140.

Arzheimer, K. (2016). Strukturgleichungsmodelle. Eine anwendungsorientierte Einführung. Wiesbaden: Springer VS.

Autorengruppe Bildungsberichterstattung (2018). Bildung in Deutschland 2018. Ein indikatorengestützter Bericht mit einer Analyse zu Bildung und Migration. Bielefeld: wbv.

Autorengruppe Bildungsberichterstattung (2020). Bildung in Deutschland 2020. Ein indikatorengestützter Bericht mit einer Analyse zu Bildung in einer digitalisierten Welt. Bielefeld: wbv.

Avramidis, E., \& Norwich, B. (2002). Teachers' attitudes towards integration/inclusion: a review of the literature. European Journal of Special Needs Education, 17(2), 129-147.

Bandura, A. (1997). Self-efficacy: the exercise of control. New York: Freeman.

Baumert, J., \& Kunter, M. (2006). Stichwort: Professionelle Kompetenz von Lehrkräften. Zeitschrift für Erziehungswissenschaft, 9(4), 469-520.

Biedermann, H., Steinmann, S., \& Oser, F. (2015). „Glaubensbestände und Glaubenswandel“. Zur Transformation von konstruktions- und transmissionsorientierten Lehr-Lern-Überzeugungen in der Lehrpersonenausbildung. Beiträge zur Lehrerinnen- und Lehrerbildung, 33(1), 46-68.

Blossfeld, H.-P., Roßbach, H.-G., \& von Maurice, J. (Hrsg.). (2011). Education as a lifelong process: the German National Educational Panel Study (NEPS) (Zeitschrift für Erziehungswissenschaft: Sonderheft 14). Wiesbaden: VS.

de Boer, A., Pijl, S. J., \& Minnaert, A. (2011). Regular primary schoolteachers' attitudes towards inclusive education: a review of the literature. International Journal of Inclusive Education, 15(3), 331-353.

Bosse, S., \& Spörer, N. (2014). Erfassung der Einstellung und der Selbstwirksamkeit von Lehramtsstudierenden zum inklusiven Unterricht. Empirische Sonderpädagogik, 6(4), 279-299.

Bosse, S., Henke, T., Jäntsch, C., Lambrecht, J., Vock, M., \& Spörer, N. (2016). Die Entwicklung der Einstellung zum inklusiven Lernen und der Selbstwirksamkeit von Grundschullehrkräften. Empirische Sonderpädagogik, 8(1), 103-116.

Bosse, S., Jäntsch, C., Henke, T., Lambrecht, J., Koch, H., \& Spörer, N. (2017). Das Zusammenspiel der Offenheit für Innovationen, der Einstellung zum inklusiven Lernen und der Selbstwirksamkeit von Lehrkräften. Zeitschrift für Bildungsforschung, 7(2), 131-146.

Boyle, C., Topping, K., \& Jindal-Snape, D. (2013). Teachers' attitudes towards inclusion in high schools. Teachers and Teaching, 19(5), 527-542.

Brouwer, N., \& Korthagen, F. (2005). Can teacher education make a difference? American Educational Research Journal, 42(1), 153-224.

Christ, O., \& Schlüter, E. (2012). Strukturgleichungsmodelle mit Mplus. Eine praktische Einführung. München: Oldenburg.

Civitillo, S., Vervloed, M.P.J., \& de Moor, J.M.H. (2016). Pre-service teachers' beliefs about inclusive education in the Netherlands: an exploratory study. Support for Learning, 31(2), 104-121.

Dicke, T., Holzberger, D., Kunina-Habenicht, O., Linninger, C., Schulze-Stocker, F., Seidel, T., Terhart, E., Leutner, D., \& Kunter, M. (2016). „Doppelter Praxisschock“ auf dem Weg ins Lehramt? Verlauf und potenzielle Einflussfaktoren emotionaler Erschöpfung während des Vorbereitungsdienstes und nach dem Berufseintritt. Psychologie in Erziehung und Unterricht, 63(4), 244-257. 
Eagly, A.H., \& Chaiken, S. (1993). The psychology of attitudes. Fort Worth: Harcourt Brace Jovanovich College Publishers.

Eichfeld, C., \& Algermissen, P. (2016). Aber nicht in Sachsen...? Synopse von Studien zu Einstellungen sächsischer Grundschullehrkräfte zu inklusivem Unterricht. In K. Liebers, B. Landwehr, S. Reinhold, S. Riegler \& R. Schmidt (Hrsg.), Facetten grundschulpädagogischer und -didaktischer Forschung (S. 67-72). Wiesbaden: Springer.

Fishbein, M. (1967). A consideration of beliefs, and their role in attitude measurement. In M. Fishbein (Hrsg.), Readings in attitude theory and measurement (S. 257-266). New York: Wiley.

Fives, H., \& Buehl, M. M. (2012). Spring cleaning for the "messy" construct of teachers' beliefs: What are they? Which have been examined? What can they tell us? In K. R. Harris, S. Graham \& T. Urdan (Hrsg.), Individual differences and cultural and contextual factors (Educational psychology handbook, Bd. 2, S. 471-499). Washington, DC: American Psychological Association.

Gebhardt, M., Kuhl, J., Wittich, C., \& Wember, F. B. (2018). Inklusives Modell in der Lehramtsausbildung nach den Anforderungen der UN-BRK. In S. Hußmann \& B. Welzel (Hrsg.), DoProfiL - Das Dortmunder Profil für inklusionsorientierte Lehrerinnen- und Lehrerbildung (S. 279-292). Münster: Waxmann.

Gebhardt, M., Schwab, S., Nusser, L., \& Hessels, M. (2015). Einstellungen und Selbstwirksamkeit von Lehrerinnen und Lehrern zur schulischen Inklusion in Deutschland - eine Analyse mit Daten des Nationalen Bildungspanels Deutschlands (NEPS). Empirische Pädagogik, 29(2), 211-229.

Greiner, F., Taskinen, P., \& Kracke, B. (2020). Einstellungen und Selbstwirksamkeitsüberzeugungen von Lehramtsstudierenden bezüglich inklusiven Unterrichts: Zusammenhänge mit Kontakterfahrungen und Grundlagenkenntnissen über schulische Inklusion. Unterrichtswissenschaft, 48(2), 273-295.

Greve, W., \& Hauenschild, K. (2017). Einstellungen zu Inklusion in der Schule - ein Schlüssel zum Gelingen einer tiefgreifenden Reform. Diskurs Kindheits- und Jugendforschung, 12(3), 313-328.

Hascher, T. (2014). Forschung zur Wirksamkeit der Lehrerbildung. In E. Terhart, H. Bennewitz \& M. Rothland (Hrsg.), Handbuch der Forschung zum Lehrerberuf (2. Aufl., S. 542-571). Münster: Waxmann.

Hecht, P., \& Weber, C. (2020). Inklusionsrelevante Selbstwirksamkeitsüberzeugungen und Einstellungen von Studierenden und Lehrkräften im Berufseinstieg - Entwicklung und Zusammenhänge im Längsschnitt. Zeitschrift für Bildungsforschung, 10(1), 23-41.

Hecht, P., Niedermair, C., \& Feyerer, E. (2016). Einstellungen und inklusionsbezogene Selbstwirksamkeitsüberzeugungen von Lehramtsstudierenden und Lehrpersonen im Berufseinstieg - Messverfahren und Befunde aus einem Mixed-Methods-Design. Empirische Sonderpädagogik, 1, 86-102.

Hellmich, F., \& Görel, G. (2014). Erklärungsfaktoren für Einstellungen von Lehrerinnen und Lehrern zum inklusiven Unterricht in der Grundschule. Zeitschrift für Bildungsforschung, 4(3), 227-240.

Hellmich, F., \& Görel, G. (2016). Erfahrungen und Selbstwirksamkeitsüberzeugungen in Bezug auf inklusiven Unterricht bei Grundschullehrkräften. In K. Liebers, B. Landwehr, S. Reinhold, S. Riegler \& R. Schmidt (Hrsg.), Facetten grundschulpädagogischer und-didaktischer Forschung (S. 81-86). Wiesbaden: Springer.

Hellmich, F., Görel, G., \& Schwab, S. (2016). Einstellungen und Motivation von Lehramtsstudentinnen und -studenten in Bezug auf den inklusiven Unterricht in der Grundschule. Ein Vergleich zwischen Deutschland und Österreich. Empirische Sonderpädagogik, 8(1), 67-85.

Hellmich, F., Löper, M.F., \& Görel, G. (2019). The role of primary school teachers' attitudes and selfefficacy beliefs for everyday practices in inclusive classrooms-a study on the verification of the 'Theory of Planned Behaviour. Journal of Research in Special Educational Needs, 19(S1), 36-48.

Helmke, A. (2009). Unterrichtsqualität und Lehrerprofessionalität. Diagnose, Evaluation und Verbesserung des Unterrichts. Seelze: Klett.

Hochschulrektorenkonferenz und Kultusministerkonferenz (2015). Lehrerbildung für eine Schule der Vielfalt. Gemeinsame Empfehlung von Hochschulrektorenkonferenz und Kultusministerkonferenz. Beschluss der Kultusministerkonferenz vom 12.03.2015/Beschluss der Hochschulrektorenkonferenz vom 18.03.2015. https://www.kmk.org/fileadmin/Dateien/veroeffentlichungen_beschluesse/2015/ 2015_03_12-Schule-der-Vielfalt.pdf. Zugegriffen: 15. Juni 2020.

Hu, L., \& Bentler, P. M. (1999). Cutoff criteria for fit indexes in covariance structure analysis. Conventional criteria versus new alternatives. Structural Equation Modeling, 6(1), 1-55.

Jordan, A., Schwartz, E., \& McGhie-Richmond, D. (2009). Preparing teachers for inclusive classrooms. Teaching and Teacher Education, 25(4), 535-542.

Junker, R., Zeuch, N., Rott, D., Henke, I., Bartsch, C., \& Kürten, R. (2020). Zur Veränderbarkeit von Heterogenitäts-Einstellungen und -Selbstwirksamkeitsüberzeugungen von Lehramtsstudierenden durch diversitätssensible hochschuldidaktische Lehrmodule. Empirische Sonderpädagogik, 12(1), 45-63. 
Kim, J.-R. (2011). Influence of teacher preparation programmes on preservice teachers' attitudes toward inclusion. International Journal of Inclusive Education, 15(3), 355-377.

Kopp, B. (2009). Inklusive Überzeugung und Selbstwirksamkeit im Umgang mit Heterogenität - Wie denken Studierende des Lehramts für Grundschulen? Empirische Sonderpädagogik, 1(1), 5-25.

Kraska, J., \& Boyle, C. (2014). Attitudes of preschool and primary school pre-service teachers towards inclusive education. Asia-Pacific Journal of Teacher Education, 42(3), 228-246.

Kultusministerkonferenz (2011). Inklusive Bildung von Kindern und Jugendlichen mit Behinderungen in Schulen. Beschluss der Kultusministerkonferenz vom 20.10.2011. https://www.kmk.org/fileadmin/ veroeffentlichungen_beschluesse/2011/2011_10_20-Inklusive-Bildung.pdf. Zugegriffen: 15. Juni 2020.

Kunter, M., \& Pohlmann, B. (2015). Lehrer. In E. Wild \& J. Möller (Hrsg.), Pädagogische Psychologie (2. Aufl., S. 261-281). Berlin: Springer.

Kunter, M., Baumert, J., Blum, W., Klusmann, U., Krauss, S., \& Neubrand, M. (Hrsg.). (2011). Professionelle Kompetenz von Lehrkräften. Ergebnisse des Forschungsprogramms COACTIV. Münster: Waxmann.

Lange, V. (2017). Inklusive Bildung in Deutschland. Ländervergleich. Gute Gesellschaft - soziale Demokratie 2017plus. Berlin. http://library.fes.de/pdf-files/studienfoerderung/13493.pdf. Zugegriffen: 25. Juni 2020.

Langner, A. (2015). Kompetent für einen inklusiven Unterricht. Eine empirische Studie zu Beliefs, Unterrichtsbereitschaft und Unterricht von LehrerInnen. Wiesbaden: Springer VS.

Laschke, C., \& König, J. (2014). Erfassung der Lerngelegenheiten (opportunities to learn, OTL). In C. Laschke \& S. Blömeke (Hrsg.), Teacher education and development study: Learning to teach mathematics (TEDS-M 2008). Dokumentation der Erhebungsinstrumente (S. 45-108). Münster: Waxmann.

Malinen, O.-P., Savolainen, H., Engelbrecht, P., Xu, J., Nel, M., Nel, N., \& Tlale, D. (2013). Exploring teacher self-efficacy for inclusive practices in three diverse countries. Teaching and Teacher Education, $33,34-44$.

Malinen, O.-P., Savolainen, H., \& Xu, J. (2012). Beijing in-service teachers' self-efficacy and attitudes towards inclusive education. Teaching and Teacher Education, 28(4), 526-534.

Messner, H., \& Reusser, K. (2000). Die berufliche Entwicklung von Lehrpersonen als lebenslanger Prozess. Beiträge zur Lehrerbildung, 18(2), 157-171.

Miesera, S. (2018). Inklusion an beruflichen Schulen - Einstellungen und Selbstwirksamkeitserwartungen von angehenden Lehrkräften im internationalen Vergleich (Dissertation). München: Technische Universität München.

Miesera, S., \& Gebhardt, M. (2018). Inclusive vocational schools in Canada and Germany. A comparison of vocational pre-service teachers' attitudes, self-efficacy and experiences towards inclusive education. European Journal of Special Needs Education, 33(5), 707-722.

Müller-Fohrbrodt, G., Cloetta, B., \& Dann, H.-D. (1978). Lehrerausbildung und-weiterbildung: Der Praxisschock bei jungen Lehrern. Formen, Ursachen, Folgerungen; eine zusammenfassende Bewertung theoretischer und empirischer Erkenntnisse. Stuttgart: Klett.

Pajares, M.F. (1992). Teachers' beliefs and educational research: cleaning up a messy construct. Review of Educational Research, 62(3), 307-332.

Pfitzner-Eden, F. (2016). I feel less confident so I quit? Do true changes in teacher self-efficacy predict changes in preservice teachers' intention to quit their teaching degree? Teaching and Teacher Education, 55, 240-254.

Richardson, V. (1996). The role of attitudes and beliefs in learning to teach. In J. P. Sikula (Hrsg.), Handbook of research on teacher education. A project of the Association of Teacher Educators (1. Aufl., S. 102-119). New York: Macmillan.

Richter, D., Kunter, M., Lüdtke, O., Klusmann, U., Anders, Y., \& Baumert, J. (2013). How different mentoring approaches affect beginning teachers' development in the first years of practice. Teaching and Teacher Education, 36, 166-177.

Ruberg, C., \& Porsch, R. (2017). Einstellungen von Lehramtsstudierenden und Lehrkräften zur schulischen Inklusion. Ein systematisches Review deutschsprachiger Forschungsarbeiten. Zeitschrift für Pädagogik, 63(4), 393-415.

Savolainen, H., Malinen, O.-P., \& Schwab, S. (2020). Teacher efficacy predicts teachers' attitudes towards inclusion-a longitudinal cross-lagged analysis. International Journal of Inclusive Education. https:// doi.org/10.1080/13603116.2020.1752826. 
Scheer, D., Scholz, M., Rank, A., \& Donie, C. (2015). Inclusive beliefs and self-efficacy concerning inclusive education among German teacher trainees and student teachers. Journal of Cognitive Education and Psychology, 14(3), 270-293.

Schmieder, L. (2019). Dynamiken der Einstellungsentwicklung. Konzeptuelle und empirische Untersuchungen am Beispiel zweier schulischer Innovationen (Dissertation). Hildesheim: UVH - Universitätsverlag Hildesheim.

Schwab, S., \& Seifert, S. (2015). Einstellungen von Lehramtsstudierenden und Pädagogikstudierenden zur schulischen Inklusion - Ergebnisse einer quantitativen Untersuchung. Zeitschrift für Bildungsforschung, 5(1), 73-87.

Schwab, S., Hellmich, F., \& Görel, G. (2017). Self-efficacy of prospective Austrian and German primary school teachers regarding the implementation of inclusive education. Journal of Research in Special Educational Needs, 17(3), 205-217.

Schwab, S., Sharma, U., \& Hoffmann, L. (2019). How inclusive are the teaching practices of my German, Maths and English teachers?-psychometric properties of a newly developed scale to assess personalisation and differentiation in teaching practices. International Journal of Inclusive Education. https:// doi.org/10.1080/13603116.2019.1629121.

Sermier Dessemontet, R., Benoit, V., \& Bless, G. (2011). Schulische Integration von Kindern mit einer geistigen Behinderung. Untersuchung der Entwicklung der Schulleistungen und der adaptiven Fähigkeiten, der Wirkung auf die Lernentwicklung der Mitschüler sowie der Lehrereinstellungen zur Integration. Emprirische Sonderpädagogik, 3(4), 291-307.

Specht, J., McGhie-Richmond, D., Loreman, T., Mirenda, P., Bennett, S., Gallagher, T., Young, G., Metsala, J., Aylward, L., \& Katz, J. (2016). Teaching in inclusive classrooms: efficacy and beliefs of Canadian preservice teachers. International Journal of Inclusive Education, 20(1), 1-15.

Steiger, J. (2019). Migrationsbedingte Heterogenität im Sportunterricht. Schüler- und fachbezogene Überzeugungen von Sport unterrichtenden Lehrpersonen. Wiesbaden: Springer VS.

Swan, B., Wolf, K., \& Cano, J. (2011). Changes in teacher self-efficacy from the student teaching experience through the third year of teaching. Journal of Agricultural Education, 52(2), 128-139.

UNESCO (1994). Die Salamanca Erklärung und der Aktionsrahmen zur Pädagogik für besondere Bedürfnisse. https://www.unesco.de/sites/default/files/2018-03/1994_salamanca-erklaerung.pdf. Zugegriffen: 8. Juni 2020.

United Nations (2006). Convention on the rights of persons with disabilities and optional protocol. https:// www.un.org/disabilities/documents/convention/convoptprot-e.pdf. Zugegriffen: 8. Juni 2020.

Universität Hildesheim (2016). Projekt E1NS. Einstellungen zu Inklusion in der Schule: Universität Hildesheim: Institut für Psychologie/Institut für Grundschuldidaktik und Sachunterricht. https://www. uni-hildesheim.de/einstellungen-inklusion-schule/. Zugegriffen: 25. Juni 2020.

Wilson, C., Woolfson, L.M., Durkin, K., \& Elliott, M. A. (2016). The impact of social cognitive and personality factors on teachers' reported inclusive behaviour. British Journal of Educational Psychology, $86(3), 461-480$.

Woolfolk Hoy, A., \& Spero, B. R. (2005). Changes in teacher efficacy during the early years of teaching: a comparison of four measures. Teaching and Teacher Education, 21(4), 343-356.

Zingg, C., \& Grob, U. (2002). Belastungswahrnehmungen von Primarlehrpersonen im Kanton Zürich beim Berufseinstieg. Beiträge zur Lehrerinnen- und Lehrerbildung, 20(2), 216-226. 